\title{
OPEN Evaluation of femtosecond laser-induced breakdown spectroscopy system as an offline coal analyzer
}

\begin{abstract}
Sahar Sheta ${ }^{1 凶}$, Zongyu Hou ${ }^{1}$, Yun Wang ${ }^{2} \&$ Zhe Wang $^{1 凶}$
Developments in femtosecond laser induced breakdown spectroscopy (fs-LIBS) applications during the last two decades have further centered on innovative métier tie-in to the advantageous properties of femtosecond laser ablation ( $f s-L A$ ) introduced into LIBS. Yet, for industrially-oriented application like coal analysis, no research has exposed to view the analytical capabilities of $\mathrm{fs}$-LA in enhancing the physical processes of coal ablation and the impact into quantitative correlation of spectra and data modeling. In a huge coal market, fast and accurate analysis of coal property is eminently important for coal pricing, combustion optimization, and pollution reduction. Moreover, there is a thirst need of precision standardization for coal analyzers in use. In this letter, the analytical performance of a one-box femtosecond laser system is evaluated relative to an industrially applied coal analyzer based on five objectives/measures: spectral correlation, relative sensitivity factors, craters topology, plasma parameters, and repeatability. Despite high-threshold operation parameters of the fs system, competitive results are achieved compared to the optimized analytical conditions of the ns-coal analyzer. Studies targeting the in-field optimization of fs-LIBS systems for coal analysis can potentially provide insights into fs-plasma hydrodynamics under harsh conditions, instrumental customization, and pave the way for a competitive next-generation of coal analyzers.
\end{abstract}

Coal energy is used to generate a remarkable chunk of the nation's electricity and clean coal industry has promoted more attention to an abundant energy source ${ }^{1,2}$. Retrofitting coal and oxy-air in a furnace for optimized combustion requisites coal handling, preparation, and power plants to extensively use quality monitoring systems. Coal quality analysis is performed by several analytical techniques which are classified into two main categories: standards and alternatives. Standard methods are a number of strictly-regulated chemical-based techniques, normally utilize a few grams of coal samples from tons travelling on a conveyor belt, and require several hours. Therefore, standard methods are not able to provide representative results due to the inhomogeneity inherent in coal nor to be qualified for real-time requirements. Alternative methods are physical-based techniques, such as: X-ray fluorescence (XRF), prompt gamma neutron activation analysis (PGNAA), laser-induced breakdown spectroscopy (LIBS), near-infrared spectrometry (NIRS), inductively-coupled plasma atomic emission spectrometry (ICP-AES), atomic absorption spectrometry (AAS), and microwave analysis. Among these, XRF, PGNAA, NIRS, and LIBS are able to provide real-time measurements. LIBS - utilizing nanosecond lasers, has been industrially applied for coal analysis with reasonably simple, robust, and compact analyzers which suit the three installation sets in a power plant: inline, at-line, and offline ${ }^{3}$. Comparatively high sensitivity (unlike XRF), safe operation (unlike PGNAA), and affordable prices (unlike NIRS) forge LIBS as a levelheaded technique for quantitative analysis in coal-fired applications. However, furtherance in measurement accuracy and performance has been one of the important concerns for wide commercialization ${ }^{4}$.

Nanosecond-based LIBS coal analyzers have a long journey with several reported letters on their designs, installation modes (in-line, at-line, and offline), and performance. Inline coal analyzers are directly shooting at coal blocks or pulverized coal over the belt with no sampling system required to feed the analyzer. This is the most convenient way for fast in-situ analysis. However, inline analyzers yield results which suffer from severe measurement errors and high uncertainty. At-line coal analyzers are placed besides the coal flow where samples

\footnotetext{
${ }^{1}$ State Key Lab of Power Systems, Department of Energy and Power Engineering, International Joint Lab on Low Carbon Clean Energy Innovation, Tsinghua University, Beijing 100084, China. ${ }^{2}$ Renewable Energy Resources Laboratory, Department of Mechanical and Aerospace Engineering, University of California, Irvine, CA 92697-3975, USA. ${ }^{\circledR}$ email: sahar_sheta@niles.edu.eg; zhewang@tsinghua.edu.cn
} 
are made using a sampling system. Offline coal analyzers are usually one-box/unit LIBS systems located near the coal utilization line or at laboratories. The sample is prepared by choosing representative samples from the belt, grinding, mixing, pressing into pellets, analyzing, and finally sending back to the coal stream. Romero et al. ${ }^{5}$ developed an offline coal analyzer to be installed in a power plant and utilized coal samples from 3 mines. Samples were crushed into $\sim 250 \mu \mathrm{m}$ and dried to remove moisture. The unit achieved measurement accuracy for elemental composition within $\pm 15 \%$ (absolute). The unit consists of a ns-ablation source (10 ns pulse width), optical spectrometer, photodiode/amplifier, and processing computer. The sample chamber, machined from aluminum, comprises a sample cart and motorized XY stage, and designed to allow a nonoxygen atmosphere. Data processing was done using artificial neural network models to determine ash fusion temperature with $\pm 14 \%{ }^{\circ} \mathrm{C}$ average precision. Zhang et al. ${ }^{6}$ designed a fully software-controlled LIBS unit with a ns-ablation source (8 ns pulse width), analysis chamber, and a control module. The analysis chamber included a stepping-motor stage and a jet pump to create negative pressure to suck the generated aerosols. A closed-loop feedback laser energy stabilization methodology reduced RSDs of energy variations from $\pm 5.2 \%$ to $\pm 1.3 \%$. Coal properties; ash content, volatile matter content, and calorific value, were predicted using SVM combined with PCA and the average relative error of prediction reduced from $8.3 \%$ to $5.48 \%, 5.83 \%$ to $4.42 \%$, and $5.4 \%$ to $3.68 \%$, respectively. On a laboratory level, Redoglio et al. ${ }^{7,8}$ designed a moving system comprised a $500 \mathrm{~mm}$-diameter circular array to house coal samples and rotate at fixed speed. The optical system with a large depth-of-field mirror was utilized to overcome the changing in coal rocks' height. This combination of sample rotating tray and optical system allowed scanning coals at desired sampling frequency to cope with the in-line requirements of analysis. A close design was employed by Gaft et al. ${ }^{9,10}$ at LDS LIBS unit (in-line mode of operation) to overcome the variation of coal height using an ultrasonic sensor which moves the entire optical system to maintain the focal length of the focusing lens. The different designs in literature have proved useful in providing technical specifications for LIBS customization in coal industry. Along with the ameliorating of data modeling, a wide research direction is opened for next generations of LIBS coal analyzers with ultrafast lasers as ablation sources.

In fact, progress in analytical techniques based on laser ablation (LA) continually places demands on developing informative analytical methods with competing performances for different applications. Ultrashort pulse lasers (pulse width $<1 \mathrm{ps}$ ) have addressed fundamental changes in the ablation process due to different mechanisms of power dissipation. LIBS, as an analytical technique widely used for solid sampling, benefits by femtosecond lasers as ablation sources and improves its figures of merit with: enhanced repeatability ${ }^{11}$, reduced matrix effects $^{12}$, better depth control and quality crater $^{13}$, and reduction in damage ${ }^{14,15}$. At present-to the authors' knowledge, only two references to date make use of fs-LIBS systems to coal analysis. Hemalaxmi et al. ${ }^{16}$ detected $\mathrm{C}, \mathrm{Al}, \mathrm{Fe}$, and $\mathrm{Ca}$ in coal and ash samples using fs-LIBS system. The carbon content was found to be correlated to the $\mathrm{C}_{2}$ and $\mathrm{CN}$ molecular bands. Jian et al. ${ }^{17}$ combined fs-LIBS with PLS model to quantitatively determine the calorific heat value in coal samples. Authors claimed that when the fs laser is used as the ablating source and a ns laser is used for heating plasma, the dual-pulse system would improve the quantification results. Despite useful results to this end, no introductory study has evaluated ultrafast lasers as innovative ablation sources for coal analysis in comparison to industrially-applied coal analyzers. This approach paves the way to methodical optimization studies from a technical point of view; necessary to provide solutions for LIBS analytical tasks in industry.

In this letter, a fs-LIBS system is evaluated in comparison to industrially applied coal analyzer. The concept described in this paper evolves in steps to cover five objectives for a performance measure that starts with emphasizing the differences between fs and ns-LAs analytical capabilities to represent coal structure by analyzing lines with highest correlation coefficients to coal properties. Molecular fragmentation and ionization are discussed to understand the high representativeness and selectiveness of lines within molecular bands correlated to coal properties in case of fs-LA. Ionic and atomic lines abundancies in fs-plasma are tested by calculating relative sensitivity factors. Coulombic regime of plasma hydrodynamics in fs-LA incurs fast dissipation of pulse energy density where electrostatic ejection and formation of atomic, ionic, and molecular fragments are dominant. Craters morphologies are examined to explain the enhanced laser-energy coupling in case of fs-LA. Plasma temperatures and electron number densities are calculated and the cooler ablation of fs plasmas is emphasized. Later, spectral repeatability is accessed by average measurement-to-measurement relative standard deviations (RSDs) of raw spectral lines. At the end of this letter, a discussion about the applicability and problems of fs-LIBS systems for industrial applications is conducted, along with the evaluation results and summary.

\section{Results and discussion}

Correlation coefficients. Direct correlation between quantitative information of coal spectra and coal analysis is one of the tentative and tricky applications of LIBS ${ }^{18}$. The situation is complicated due to: matrix complexity and property interdiscursivity of coal. The heterogeneous composition of coal, with a mixture of organic and inorganic molecules which are considerably varying in size and structure ${ }^{19}$, implements fluctuated plasma kinetics and spectral interdependency. The coal properties interdiscursivity is simply noticed by calorific heat value, volatile, and ash contents definitions. Caloric heat value is the net heat released from coal combustion with oxygen. It integrates the combustion heat of $\mathrm{C}, \mathrm{H}, \mathrm{O}, \mathrm{S}$, ash, and volatile matter contents. Volatile matter includes long-chains hydrocarbons, aromatic hydrocarbons, and sulfur. Ash includes the noncombustible residue of mineral oxides and sulfates. Therefore, major and mineral lines carry spectral information related to different coal properties. In this work, a total of 998 and 900 lines are observed by the fs and ns LIBS systems, respectively (denoted as $f$-Solstice and $n s$-Chem-see materials and systems section for more details). The congestion of the coal spectra due to abundance of mineral elements makes emission lines identification a cumbersome task and misidentification of lines is easily probable. Therefore, spectra from $f_{s}$-Solstice and $n s$-Chem systems were carefully identified using NIST database ${ }^{20}$ and confirmed by literature. A plentiful population of atomic, ionic, and 


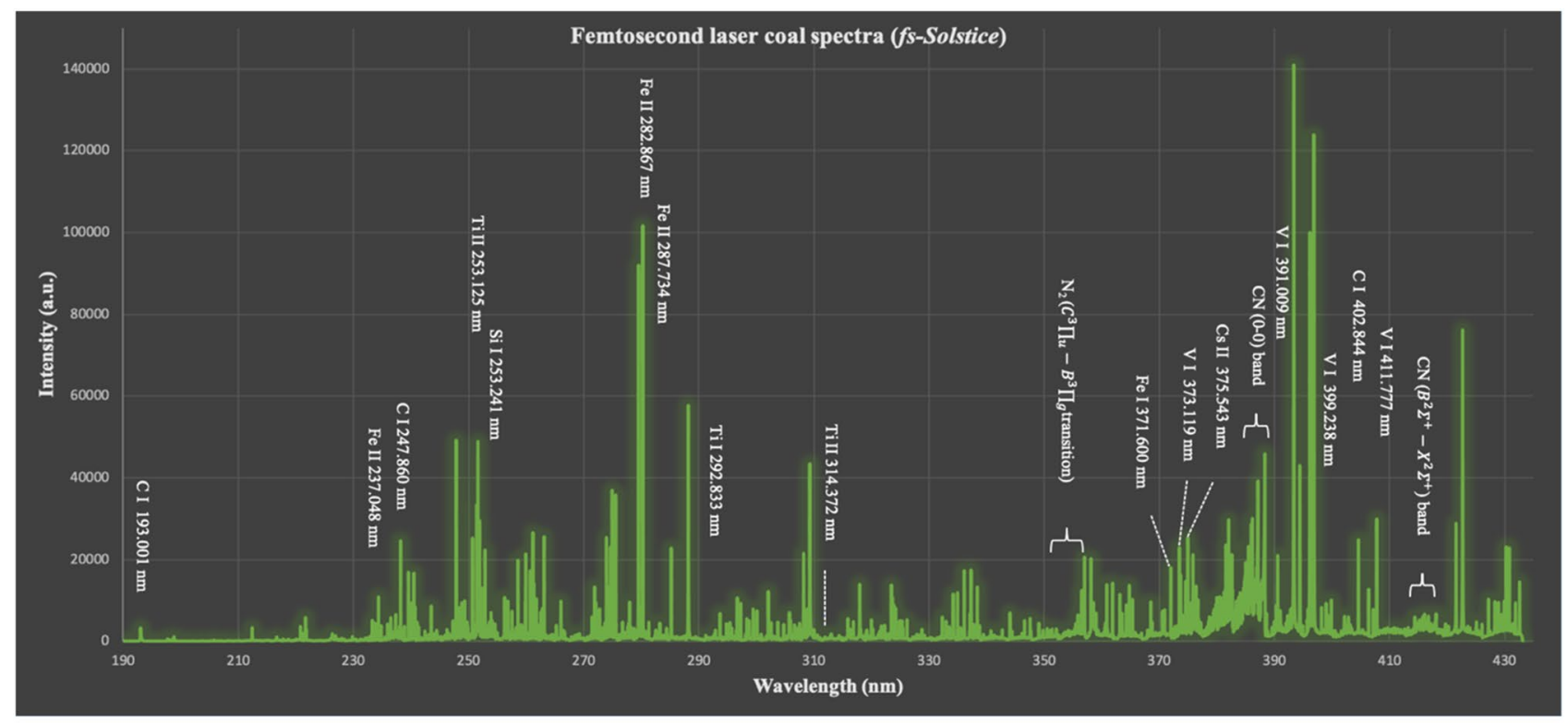

(a)

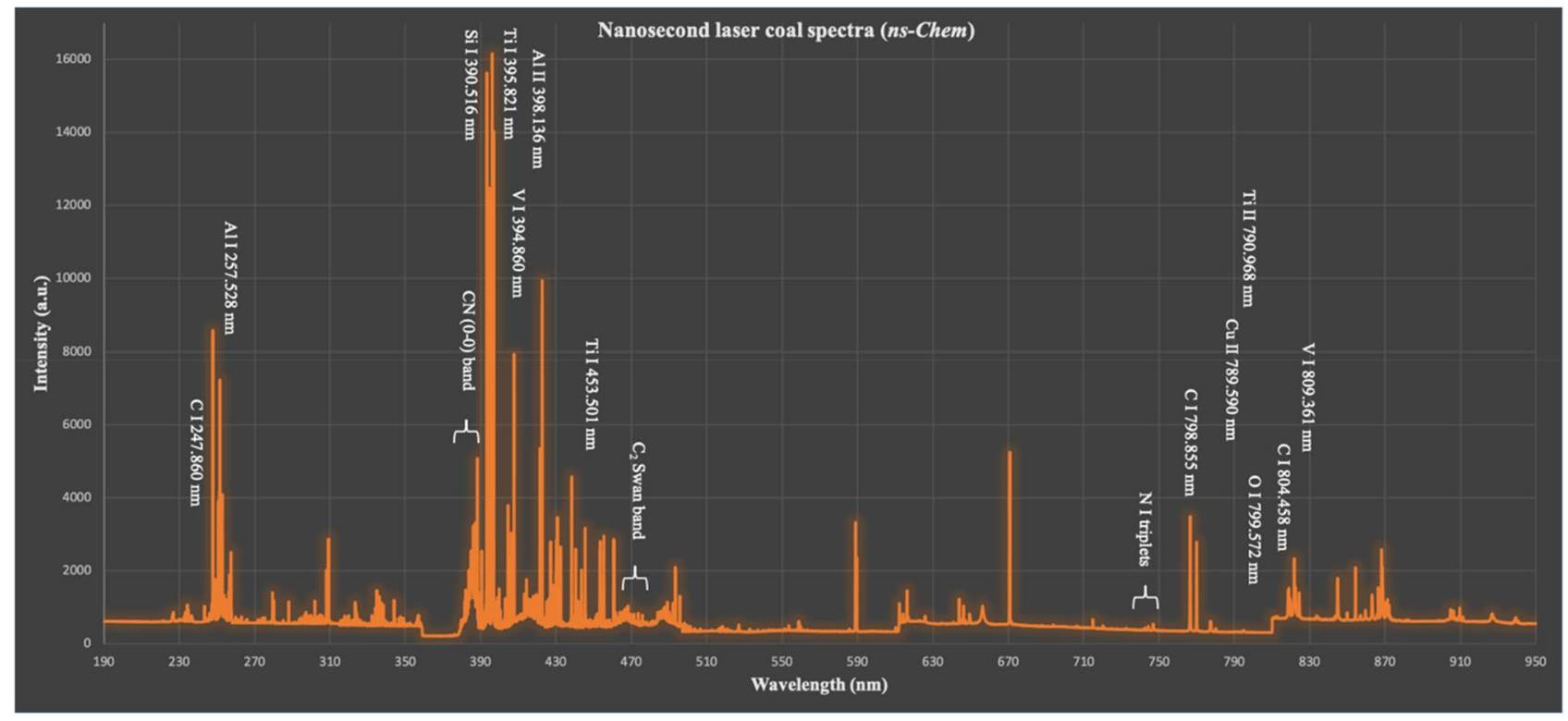

(b)

Figure 1. Coal spectra obtained by $f$ s-Solstice system in (a) and $n s$-Chem system in (b). A plentiful population of atomic, ionic, and molecular lines in both spectra indicates the enrichment of plasma species in the fs and ns ablation conditions.

molecular lines are observed in both spectra indicating the enrichment of plasma species in fs and ns ablation conditions as shown in Fig. 1.

This expected congestion of coal spectra means that the normal situation of extracting coal properties from specified spectral lines might limit the analysis accuracy due to stepwise correlations ${ }^{21}$. Consequently, several multivariate and hybrid models ${ }^{22-24}$ have been used to extract correlated spectral information to concentrations. These models, still and all, provide robust algorithms to enhance quantitative measurements for a number of applications, but have degraded accuracy for materials with heterogenous/matrix composition like coals ${ }^{3}$. With increasing chemical complexity, algorithms functionalize with solely statistical correlations or curve overfitting eventually ruin the measurement trueness due to lack of physical picture of the fingerprint emissions from parent molecules in the LA process. To this extent, it is significant to emphasize the analytical capabilities of fs-LA to represent coal structure by analyzing lines with highest correlation coefficients to coal properties relative to those obtained by the optimized $n s$-Chem system. Pearson correlation $(\boldsymbol{r})$ is commonly used in linear regression to measure a relationship strength between two variables. It is given by the formula ${ }^{25}$ : 


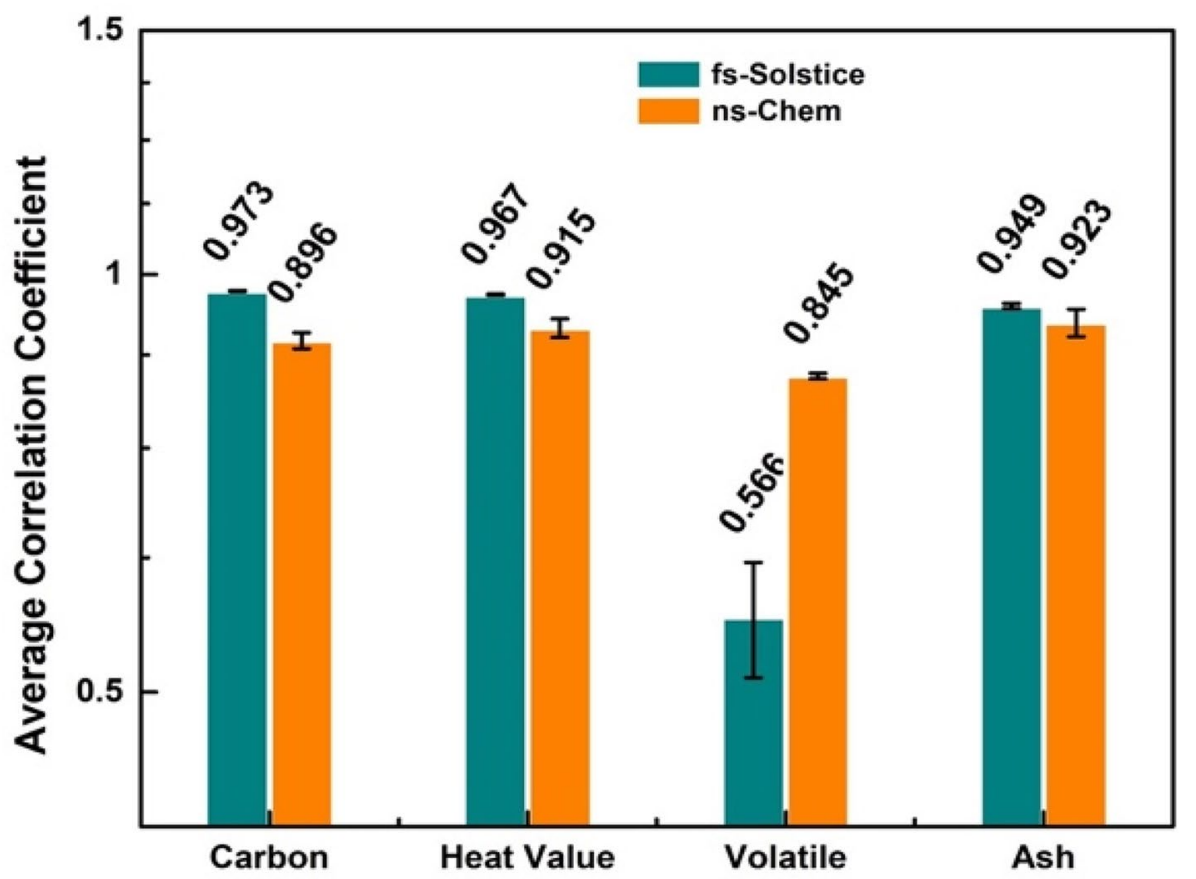

Figure 2. Average of Pearson's correlation coefficients of 10 most correlated lines to carbon content, heat value, volatile, and ash contents obtained by $f$ s-Solstice and $n s$-Chem systems.

$$
\boldsymbol{r}=\frac{\sum(x-\bar{x})(y-\bar{y})}{\sqrt{\sum(x-\bar{x})^{2}(y-\bar{y})^{2}}}
$$

where; $\boldsymbol{r}$ is the correlation coefficient, $x$ is the standard value and $y$ is the line intensity. The bar sign shows the average value of all measurements.

Pearson correlation analysis has been used for the identification, classification, and determination of physical/ chemical properties of different materials relative to reference spectra. However, to ensure correct and fair correlation magnitude of $\boldsymbol{r}$, a few factors have to be considered: (1) Essential spectra related to laser-matter interaction and probably to a surrounding gas have to be included while non-essential spectral information (random noise) have to be excluded; (2) Sufficient number of observations (well-resolved and non-saturated lines) should be available in correlated spectra to confirm correct representativeness of $\boldsymbol{r}$; (3) Multiple sampling is essential to avoid misleading $\boldsymbol{r}$ values due to strongly nonlinear character of a combination of factors such as the laser-matter interaction, surface roughness, sample heterogeneity, etc ${ }^{26}$. Considering those factors, Gornushkin et al. ${ }^{27}$ used Pearson correlation for the identification of glass samples of forensic interest. The spectral data was utilized after two pre-processing steps of: rejection of spectral outliners (filtering) and removal of spectral fragments which contain no correlated information (masking). The proposed procedure showed $100 \%$ identification rate. Lentjes et $\mathrm{al}^{28}$ found that averaging the spectra enhanced the correlation accuracy from $79 \%$ for single shot to $99.9 \%$ for average spectra. Therefore, during our work, multi-pulse averaging, background subtraction, and normalization by the whole spectral area were applied as pre-processing methods to compensate for variations in laser energies and to ensure fair $\boldsymbol{r}$ values for each system. Visual inspection of spectral lines was done to ensure that the lines which showed highest correlation are non-saturated lines, well-resolved, and have stable intensity over the 40 samples (validity for analytical use). Figure 2 shows average correlation coefficients of 10 most correlated lines for carbon content, heat value, volatile, and ash contents using $f s$-Solstice and $n s$-Chem systems. The error bars are standard deviations of 40 measurements corresponding to 40 samples in use. The correlation coefficients of carbon, heat value and ash are $\geq 0.949$ for $f$ s-Solstice and $\leq 0.923$ for $n s$-Chem showing higher representativeness of the fs spectra to coal property. For volatile matter content, the fs-spectra show correlation of 0.566 which is lower than 0.845 for the ns-spectra. The identification of the correlated lines for each coal property may uncover reasons behind low $\boldsymbol{r}$ values in case of volatile matter content and reveal differences in ablation mechanisms for $f s$-Solstice relative to $n s$-Chem.

Table 1 illustrates the identification and evaluation of the 10 most correlated lines for carbon content. The correlation coefficients vary between 0.975 and 0.971 for $f s$-Solstice, while for $n s$-Chem, correlation coefficients show larger variations between 0.907 and 0.879 for the 10 most correlated lines. For $f s$-Solstice spectra, seven lines are identified ${ }^{29}$ within the $\mathrm{CN}\left(\mathrm{B}^{2} \Sigma^{+}-\mathrm{X}^{2} \Sigma^{+}\right)$violet system $(\Delta v=-1)$, while three lines are Fe I and V I mineral lines. For $n s$-Chem spectra, two C I lines at 798.8 and $804.4 \mathrm{~nm}$ show high correlation with carbon content. Other organic lines are an O I line at 799.5 and N I triplet at 742.4, 744.3, and $746.9 \mathrm{~nm}$. Residuals are four mineral lines of V I, Cu II, Ti II, and Cs II. Same lines with different correlations order show $\boldsymbol{r}$ values ranged 


\begin{tabular}{|c|c|c|c|c|c|c|}
\hline \multicolumn{6}{|c|}{ Carbon content } & \multirow[b]{3}{*}{ Evaluation } \\
\hline \multicolumn{3}{|c|}{ fs-Solstice } & \multicolumn{3}{|c|}{ ns-Chem } & \\
\hline$\lambda / \mathbf{n m}$ & $r$ & Line & $\lambda / \mathbf{n m}$ & $r$ & Line & \\
\hline 417.645 & 0.975 & $\mathrm{CN}$ & 798.855 & 0.907 & C I & \multirow{10}{*}{ Higher representativeness of $f_{s-\text { Solstice spectra (larger } r \text { values) }}$} \\
\hline 417.953 & 0.974 & $\mathrm{CN}$ & 804.458 & 0.906 & C I & \\
\hline 371.600 & 0.974 & Fe I & 809.361 & 0.904 & V I & \\
\hline 417.526 & 0.973 & $\mathrm{CN}$ & 799.572 & 0.904 & O I & \\
\hline 418.240 & 0.973 & $\mathrm{CN}$ & 789.590 & 0.903 & $\mathrm{Cu}$ II & \\
\hline 417.189 & 0.973 & $\mathrm{CN}$ & 790.968 & 0.903 & Ti II & \\
\hline 415.808 & 0.972 & $\mathrm{CN}$ & 793.434 & \begin{tabular}{|l|}
0.902 \\
\end{tabular} & Cs II & \\
\hline 411.777 & 0.972 & V I & 744.322 & 0.882 & $\mathrm{NI}$ & \\
\hline 415.709 & 0.972 & $\mathrm{CN}$ & 742.480 & 0.881 & N I & \\
\hline 373.119 & 0.971 & V I & 746.971 & 0.879 & N I & \\
\hline
\end{tabular}

Table 1. Evaluation of $f$ s-Solstice spectra correlation to carbon content in coal samples.

\begin{tabular}{|c|c|c|c|c|c|c|}
\hline \multicolumn{6}{|c|}{ Heat value } & \multirow[b]{3}{*}{ Evaluation } \\
\hline \multicolumn{3}{|c|}{ fs-Solstice } & \multicolumn{3}{|l|}{ ns-Chem } & \\
\hline$\lambda / \mathbf{n m}$ & $r$ & Line & $\lambda / \mathbf{n m}$ & $r$ & Line & \\
\hline 415.808 & 0.970 & $\mathrm{CN}$ & 798.855 & 0.924 & C I & \multirow{10}{*}{ Higher selectiveness of $f s$-Solstice spectra (different molecular fragments correlated) } \\
\hline 375.543 & 0.968 & Cs II & 789.590 & 0.924 & $\mathrm{Cu}$ II & \\
\hline 415.236 & 0.968 & $\mathrm{CN}$ & 809.361 & 0.923 & V I & \\
\hline 356.533 & 0.968 & $\mathrm{~N}_{2}^{+}$ & 804.458 & 0.923 & $\mathrm{CI}$ & \\
\hline 355.280 & 0.968 & $\mathrm{~N}_{2}^{+}$ & 790.968 & 0.923 & Ti II & \\
\hline 354.413 & 0.968 & $\mathrm{~N}_{2}^{+}$ & 793.434 & 0.921 & Cs II & \\
\hline 373.119 & 0.968 & V I & 799.572 & 0.921 & O I & \\
\hline 418.240 & 0.968 & $\mathrm{CN}$ & 744.322 & 0.896 & N I & \\
\hline 356.204 & 0.968 & $\mathrm{~N}_{2}^{+}$ & 746.971 & 0.894 & N I & \\
\hline 415.674 & 0.967 & $\mathrm{CN}$ & 742.480 & 0.894 & N I & \\
\hline
\end{tabular}

Table 2. Evaluation of $f_{s}$-Solstice spectra correlation to heat value in coal samples.

between 0.924 and 0.894 to caloric heat content as shown in Table 2 . While for the $f s$-Solstice spectra, four lines are identified within the $\mathrm{CN}$ molecular band where two of them correlate to heat value and not to carbon content showing higher selectiveness of the fs-LA relative to its ns-counterpart. Another four lines are unforeseen emissions from the second positive band system ${ }^{30,31}\left(\mathrm{C}^{3} \prod_{u}-\mathrm{B}^{3} \prod_{g}\right.$ transition) of $\mathrm{N}_{2}$. It means that $7 / 10$ and $8 / 10$ of the most correlated lines for carbon content and heat value respectively are molecular lines for $f$ s-Solstice spectra.

During the last few years, molecular fragmentation and ionization have been evolved significantly in molecular sensing studies as fundamental interaction processes in $\mathrm{fs}-\mathrm{LA}^{32,33}$ of organic materials. Due to the short-time interaction of super intense laser fields, the geometrical structure of molecules becomes deformed within $\sim 100 \mathrm{fs}$ and tunneling ionization occurs. Charged molecules are formed triggered by strong Coulombic repulsive force and abrupt chemical bond fission occurs where atomic and ionic species with large kinetic energies are ejected. In collisional environment like air, resultant ions/fragments undergo random scattering/collision reactions in a relatively slower time scale leading to optical emissions. In an ideal reaction process; i.e. collision-less, fs-LA molecular ejecta is typically fingerprinting the parent molecules. The formation pathways of $\mathrm{CN}$ molecular bands in fs-based interaction zone have been discussed under three major formation mechanisms: direct fragmentation from species containing $\mathrm{CN}$ bonds in its structure at early stages of plasma evolution near surface (native $\mathrm{CN}$ ), recombination of atoms from compounds in plasma to produce $\mathrm{CN}$ molecules (collisions- $\mathrm{CN}$ ), and reactive atomic recombination of $\mathrm{C}$ from plasma with $\mathrm{N}$ from ambient or air (ambient-CN). For the $2^{\text {nd }}$ and $3^{\text {rd }}$ pathways, CN molecules' detection is sensitive to spatiotemporal conditions of the plasma. The persistence of $\mathrm{CN}$ molecular bands in fs-LAs is well reported. Baudelet et al. ${ }^{34}$ investigated native $\mathrm{CN}$ molecular bands of bacteria where the band-head intensity reached maximum at $450 \mathrm{~ns}$ delay time. Zhao et al. ${ }^{35}$ found that the native $\mathrm{CN}$ band-head persisted before 700-800 ns delay time (the delay time chosen in our experiment is $400 \mathrm{~ns}$ ). As for the nitrogen band, the $\mathrm{N}_{2}{ }^{+}$fragments could come from ${ }^{36}$ : collision of resultant fragments ( $\mathrm{NH}$ radicals) within the dense plasma or with ambient particles or with energetic electrons. Since N-N bond structure might not exist in coals, $\mathrm{N}_{2}{ }^{+}$fragments are probably formed due to ionization of air by energetic electrons at the interaction zone on coal surface-which is the claim behind enhanced residual thermal energy deposition explained later in this paper. Yet, the dynamical reactions of hydrocarbons in air are still under investigation ${ }^{37}$. Figure 3 shows the $\mathrm{CN}\left(\mathrm{B}^{2} \Sigma^{+}-\mathrm{X}^{2} \Sigma^{+}\right)$and the $\mathrm{N}_{2}\left(\mathrm{C}^{3} \prod_{u}-\mathrm{B}^{3} \prod_{g}\right)$ molecular systems detected into $f$ s-Solstice spectra of coal and show high correlations to carbon content and heat value. 


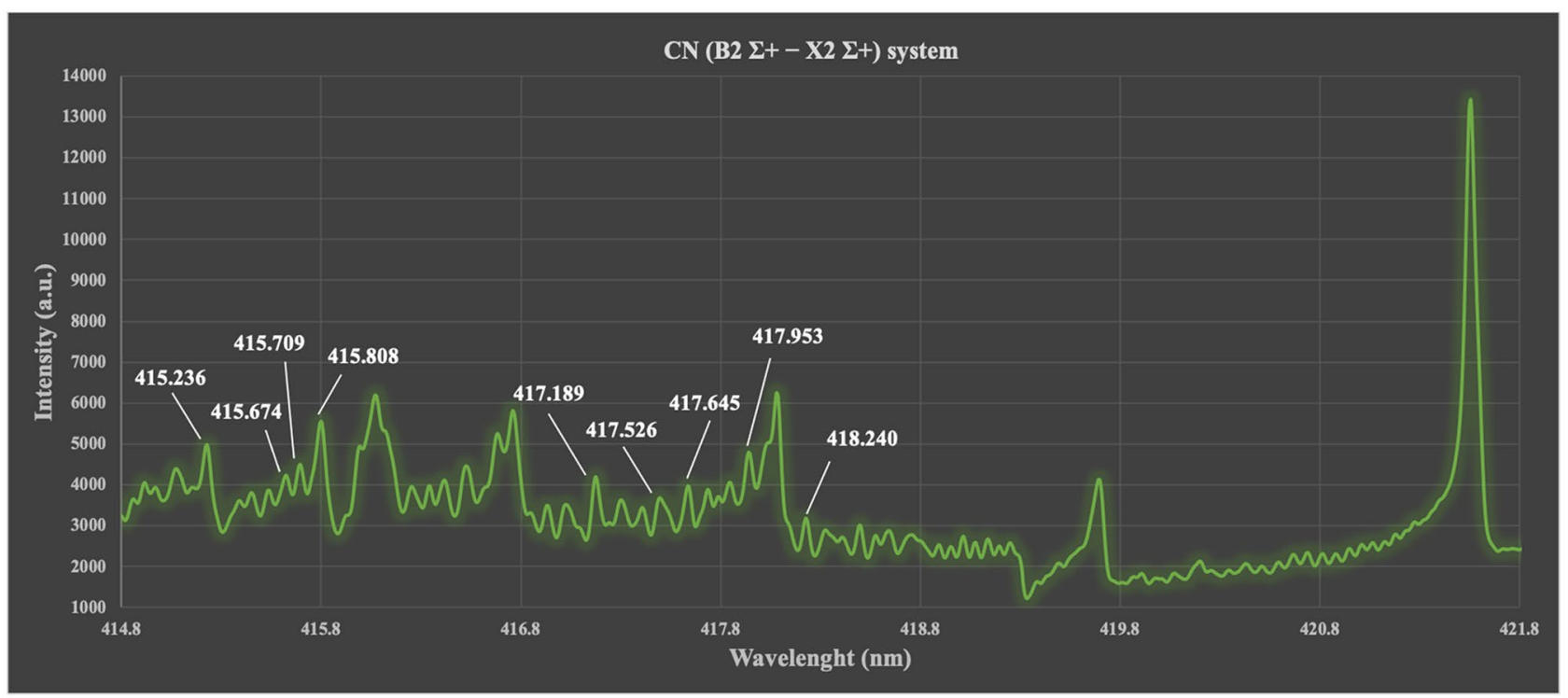

(a)

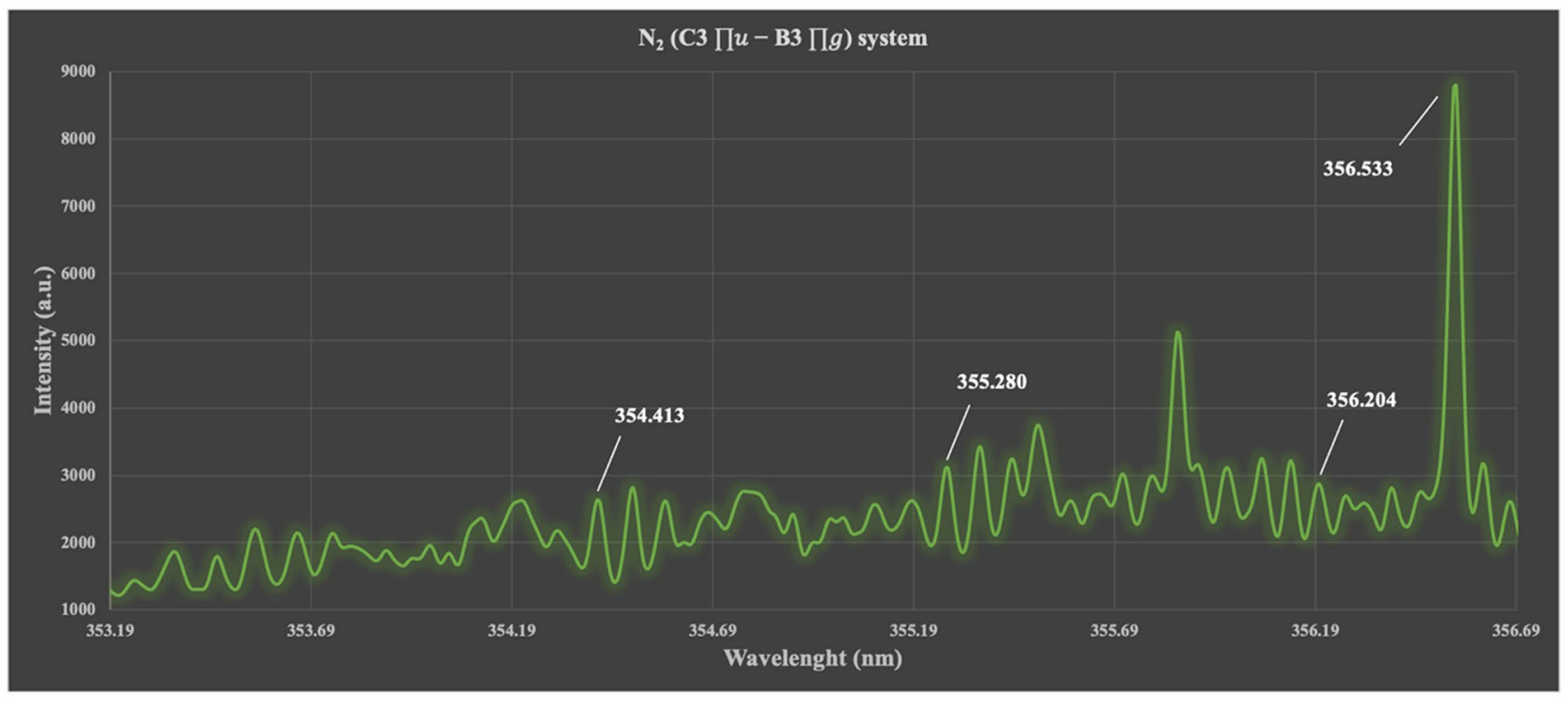

(b)

Figure 3. The $\mathrm{CN}\left(\mathrm{B}^{2} \Sigma^{+}-\mathrm{X}^{2} \Sigma^{+}\right)$and the $\mathrm{N}_{2}\left(\mathrm{C}^{3} \prod_{u}-\mathrm{B}^{3} \prod_{g}\right)$ molecular systems detected into $f$ s-Solstice spectra of coal and lines within which show high correlations to carbon content and/or heat value.

As for volatile matter content, Table 3 depicts the lines of $f s$-Solstice and $n s$-Chem spectra which show highest correlations to volatile matter content. For $n s$-Chem spectra, lines are detected within the $\mathrm{C}_{2}$ swan system $\left(d^{3} \prod_{g}-a^{3} \prod_{u}\right)$ and $\boldsymbol{r}$ values are in the range $0.853-0.841$ (weak correlation values). As a consensus reality, volatile matter content determination is a hectic task due to limitations related to the accuracy of the eventual detection of elements integrating spectral information to the property content. In a sense, volatile matter is standardly measured as the weight percent of gas (emissions) that is released during heating a dried coal sample to $950{ }^{\circ} \mathrm{C}$ in an oxygen-free environment by thermogravimetric analysis (TGA). The integrated intensities of $\mathrm{C}, \mathrm{H}, \mathrm{O}, \mathrm{N}$, and $\mathrm{S}$ lines are hypothetically representing correlated data set to volatile content. However, $\mathrm{O}$ and $\mathrm{N}$ can be originating from the surrounding air and reaction of $\mathrm{O}$ with minerals in coal to form ash have to be considered during data handling. Moreover, sulfur is volatilized as sulfur dioxide with hardly detectable lines in many experimental protocols. Yao et al. ${ }^{38}$ discussed coals with different volatile contents and found that changes in volatile content ushered different emission line intensities and spatial distribution of major and mineral elements in the evolved ns-plasma. Several experimental and data modeling methods have been discussed in literature to enhance volatile matter content measurement by LIBS. We refer to the collection of works summarized in the analytical results section of the tutorial review by Sheta et al. ${ }^{3}$. For $f s$-Solstice spectra, weaker correlation is detected ( $r$ values are in the range 0.704-0.518) than those of the reference spectral set. Despite $6 / 10$ of the most correlated lines are $\mathrm{CN}$ and $\mathrm{C}$ I lines, it may just be a statistical artifact instead of based on real physical 


\begin{tabular}{|c|c|c|c|c|c|c|}
\hline \multicolumn{6}{|c|}{ Volatile content } & \multirow[b]{3}{*}{ Evaluation } \\
\hline \multicolumn{3}{|c|}{ fs-Solstice } & \multicolumn{3}{|c|}{ ns-Chem } & \\
\hline$\lambda / \mathbf{n m}$ & $r$ & Line & $\lambda / \mathrm{nm}$ & $r$ & Line & \\
\hline 314.372 & 0.704 & Ac I & 473.599 & 0.853 & $\mathrm{C}_{2}$ & \multirow{10}{*}{ Weak correlation of both $f_{s}$-Solstice and $n s$-Chem (reference data set) spectra } \\
\hline 387.646 & 0.585 & $\mathrm{CN}$ & 471.403 & 0.848 & $\mathrm{C}_{2}$ & \\
\hline 387.692 & 0.575 & $\mathrm{CN}$ & 468.383 & 0.846 & $\mathrm{C}_{2}$ & \\
\hline 391.009 & 0.573 & V I & 470.332 & 0.845 & $\mathrm{C}_{2}$ & \\
\hline 387.588 & 0.567 & $\mathrm{CN}$ & 470.131 & 0.844 & $\mathrm{C}_{2}$ & \\
\hline 399.238 & 0.549 & V I & 469.662 & 0.844 & $\mathrm{C}_{2}$ & \\
\hline 402.844 & 0.535 & C I & 470.667 & 0.842 & $\mathrm{C}_{2}$ & \\
\hline 373.964 & 0.526 & $\mathrm{~Pb} \mathrm{I}$ & 470.466 & 0.842 & $\mathrm{C}_{2}$ & \\
\hline 387.744 & 0.524 & $\mathrm{CN}$ & 471.002 & 0.841 & $\mathrm{C}_{2}$ & \\
\hline 193.001 & 0.518 & C I & 469.997 & 0.841 & $\mathrm{C}_{2}$ & \\
\hline
\end{tabular}

Table 3. Evaluation of $f$ s-Solstice spectra correlation to volatile content in coal samples.

\begin{tabular}{|c|c|c|c|c|c|c|}
\hline \multicolumn{6}{|c|}{ Ash content } & \multirow[b]{3}{*}{ Evaluation } \\
\hline \multicolumn{3}{|c|}{ fs-Solstice } & \multicolumn{3}{|c|}{ ns-Chem } & \\
\hline$\lambda / \mathbf{n m}$ & $r$ & Line & $\lambda / \mathbf{n m}$ & $r$ & Line & \\
\hline 253.125 & 0.955 & Ti II & 395.821 & 0.949 & Ti I & \multirow{10}{*}{ High correlation of both $f_{s}$-Solstice and $n s$-Chem spectra } \\
\hline 292.833 & 0.954 & Ti I & 395.661 & 0.947 & Fe I & \\
\hline 237.048 & 0.952 & Fe II & 394.860 & 0.943 & V I & \\
\hline 282.867 & 0.950 & Fe II & 399.886 & 0.929 & Ti I & \\
\hline 287.734 & 0.950 & Fe I & 398.136 & 0.927 & $\mathrm{Al} \mathrm{II}$ & \\
\hline 237.517 & \begin{tabular}{|l|}
0.948 \\
\end{tabular} & Fe II & 398.933 & 0.922 & Ti I & \\
\hline 226.005 & 0.947 & Fe II & 453.501 & 0.916 & Ti I & \\
\hline 236.923 & \begin{tabular}{|l|}
0.945 \\
\end{tabular} & Fe II & 453.289 & 0.910 & Ti I & \\
\hline 309.712 & 0.943 & Ti II & 390.516 & 0.896 & Si I & \\
\hline 253.241 & 0.943 & Si I & 257.528 & 0.885 & $\mathrm{Al} \mathrm{I}$ & \\
\hline
\end{tabular}

Table 4. Evaluation of $f s$-Solstice spectra correlation to ash content in coal samples.

background. Worth to mention that the molecular band correlated to volatile content by the $n s$-Chem spectra is out of the spectrometer range for the $f$ s-Solstice system. The importance of establishing an accurate correlation between spectral data set and volatile matter content urges attention to further studies based on understandable interpretation of LA-physics. For ash content, Table 4 collects the identified lines correlated to the property. Both spectra revealed well-resolved, non-saturated, and mineral lines of $\mathrm{Ti}$, Si, and $\mathrm{Fe}$ with larger $\boldsymbol{r}$ values detected for $f s$-Solstice lines. Unexpectedly, mineral lines most correlated to ash content are mainly ionic for $f$ s-Solstice spectra and atomic for $n s$-Chem which is contrast to the claim of fs-plasmas atomic nature ${ }^{39,40}$. The evaluation of atomic and ionic lines abundance in $f$ s-Solstice spectra would be investigated in the next performance measure of the conducted evaluation study.

Relative sensitivity factor. With a few exceptions, minerals are the prim hosts of vast majority of elements in coals ${ }^{41}$. On the basis of their concentration, mineral elements are considered minor and trace elements. However, they are easily atomized and ionized in coal matrix like $\mathrm{Fe}, \mathrm{Si}, \mathrm{Mg}, \mathrm{Al}, \mathrm{Ti}$, and $\mathrm{Ca}$ and attribute to higher emissions from matrixes and early stages of plasma formation ${ }^{42}$. Mineral elements affect, straightforwardly, the detection accuracy of volatile and ash contents, and the plethora of their lines in coal spectra plays a key role in full spectral information applied in several multivariate algorithms for enhanced quantitation. Relative sensitivity factor (RSF) is used to measure the spectral detection discernment of an element by intensity/concentration ratios normalized to an internal standard element. In other words, RSFs correct for the differences in the response to several elements by a specific technique, as well as for changes in the response to a species due to changes in matrix. A wide variety of analytical techniques, viz. glow discharge mass spectroscopy ${ }^{43}$ and secondary ion mass spectrometry ${ }^{44}$, rely on the use of RSFs for obtaining semi-quantitative results for multicomponent samples. In this work, RSFs have been determined for the analysis of 6 mineral elements from multiple analyses of the 40 standard coal samples representing different matrices for $f$ s-Solstice system relative to $n s$-Chem system. As each element has a fixed concentration in a specific sample, the sensitivity response of the fs system is measured with RSF, which is determined as follows ${ }^{45}$ :

$$
\mathrm{RSF}=\left(\mathrm{I}_{\mathrm{x}} / \mathrm{I}_{\mathrm{M}}\right)_{\mathrm{fs}} /\left(\mathrm{I}_{\mathrm{x}} / \mathrm{I}_{\mathrm{M}}\right)_{\mathrm{ns}}
$$




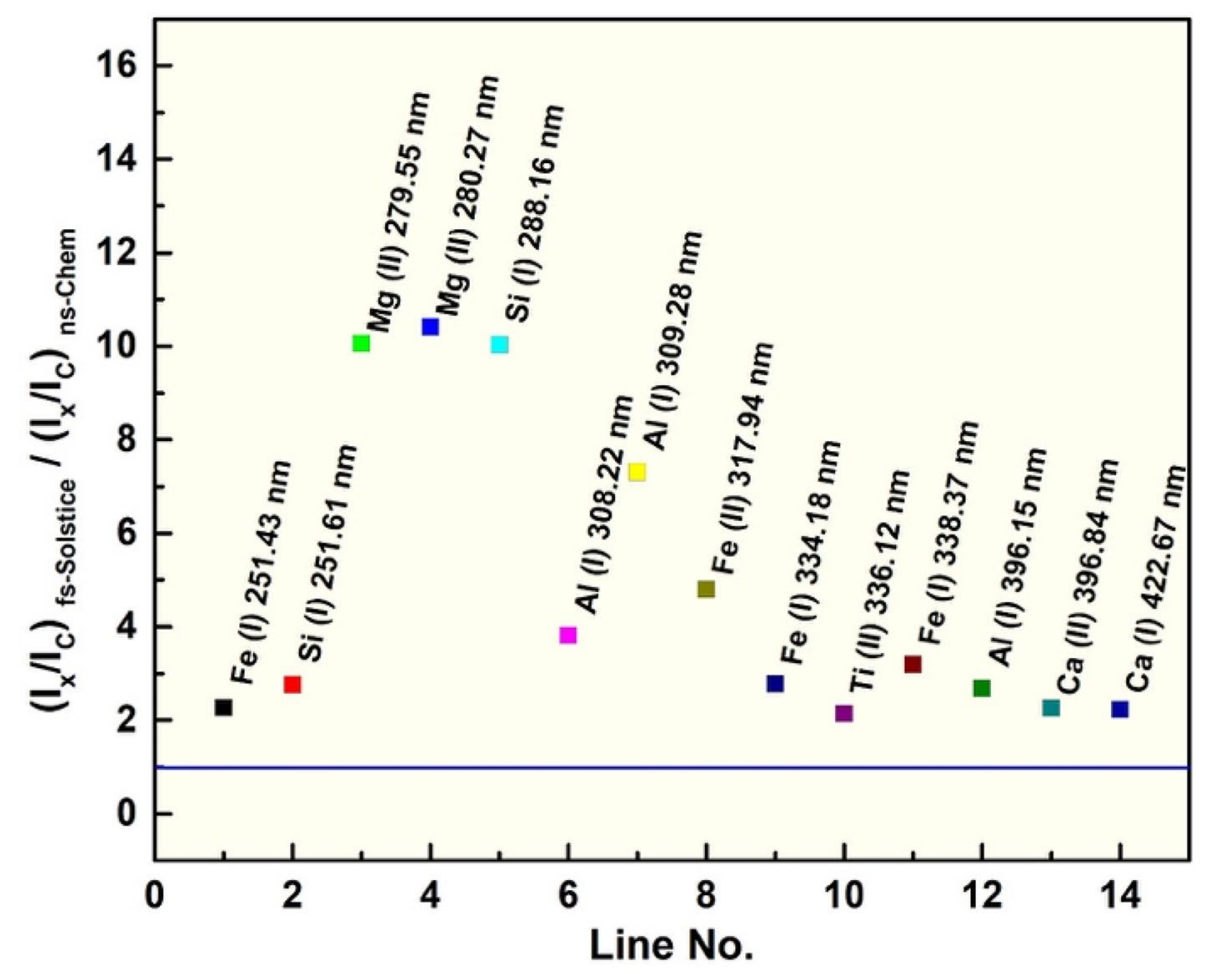

Figure 4. Relative sensitivity factors for 6 mineral elements: $\mathrm{Fe}, \mathrm{Si}, \mathrm{Mg}, \mathrm{Al}, \mathrm{Ti}$, and $\mathrm{Ca}$ in coal matrix vary between 2 to 10 showing enhanced sensitivity of $f s$-Solstice system relative $n s$-Chem system for minerals detection.

where; $\mathrm{x}$ is the element of interest and $\mathrm{M}$ is an internal standard element. Since carbon is presented in all coal samples, it was chosen as the internal standard element for RSF calculations.

Figure 4 depicts the mean RSFs for $\mathrm{Fe}, \mathrm{Si}, \mathrm{Mg}, \mathrm{Al}, \mathrm{Ti}$, and $\mathrm{Ca}$ elements in 40 coal samples and are higher than 1 for all elements. RSFs vary between 2 and 10, showing enhanced detection sensitivity for $f$ s-Solstice system relative to its ns counterpart. For prominent lines such as Mg II $279.55 \mathrm{~nm}, \mathrm{Mg}$ II $280.27 \mathrm{~nm}$ (commonly used for plasma temperature correction to cancel its dependence on line intensities ${ }^{46}$ ), and Si I $288.16 \mathrm{~nm}$ (commonly used as internal standard), RSFs are $\sim 10$ indicating 10 times enhanced detection of those lines in a normalized spectra to its dominant element, i.e. carbon to by $f s$-Solstice system. Meanwhile, the response factor for $\mathrm{Fe}, \mathrm{Al}, \mathrm{Ti}$, and Ca elements shows RSFs between 2 and 7. Gross et al. ${ }^{47}$ correlated the RSFs of 4 alkali metals in single-particle detection to both the ionization potential and lattice energy. A matter of fact is that for multicomponent analysis, factors such as the atomization/ionization energy, lattice energy, absorption cross-section at the ionization wavelength, and laser-particles interaction-all vary within each plasma and contribute to the final discernment in each system. Zhang et al. ${ }^{12}$ demonstrated that the discrepancy of RSFs between fs and ns LAs is attributed to the fundamental basis of high-irradiance laser ionization process in case of fs ablation. The RSFs variations reduced to half for the fs system relative to ns-laser mode indicating reduction in matrix-effects. Owing to the ultra-intense laser irradiance of fs laser ablation, atoms in lattice are atomized and ionized through multiphoton ionization, tunnel ionization, electron impact ionization, and avalanche ionization leading to a metastable plasma cloud with direct ejection of ions in the solid-gas-mixture phase ${ }^{48}$. The higher RSFs for elemental detection makes $f$ s-Solstice system a competitive choice for industrial coal quality analysis applications, and indeed, for monitoring environmental pollutants of coal combustion reactions. Moreover, significantly high response factors for mineral elements adds a one-point advantage for $f s$-Solstice system far beyond $n s$-Chem system for the detection of minor and trace elements compared to x-ray diffraction (XRD) which provides little information on trace elements associated with coal minerals.

The RSFs of ionic-to-atomic and ionic-to-ionic lines within $f s$-Solstice spectra are further studied to investigate the abundance of ionic lines in fs plasma and their persistence in different spectra (40 spectra correlating to 40 samples in use) - see Fig. 5. The RSFs of Ca II $393.36 \mathrm{~nm}$ to Al I $394.40 \mathrm{~nm}$ lines show variations around 2 with average RSF of $\sim 2 \pm 0.5$ for all samples. This indicates enhanced presence/abundance of ionic lines in fs plasmas. While, the average RSF of Mg II $280.27 \mathrm{~nm}$ to $\mathrm{Mg}$ II $279.55 \mathrm{~nm}$ has a mean value of $\sim 1.02 \pm 0.04$ showing similar presence for the same transition states. It is important to note that both of the selected transition sets are closely spaced to avoid the detector sensitivity issues for further apart transitions in the optical spectrum. The plentiful population of both atomic and ionic lines in fs coal plasma is in contrast to several research work, in which dominancy of atomic lines was reported. Freeman et al. ${ }^{39}$ demonstrated neutral emission with little-to-no ionic emission from fs-plasma of brass sample in both vacuum and atmospheric conditions. LaHaye et al. ${ }^{40}$ claimed fs-LA plume to be more atomic due to absence of laser-plasma coupling relative to the more ionized plume of 


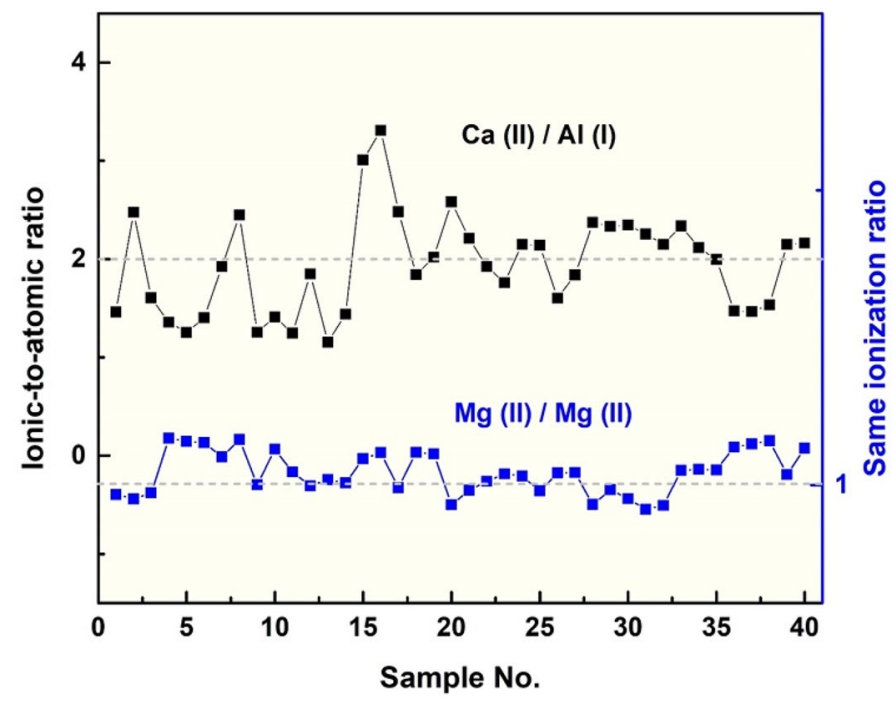

Figure 5. Relative sensitivity factors of ionic-to-atomic and ionic-to-ionic lines detected within $f$ s-Solstice spectra proving abundant presence of ionic lines in fs plasmas.

ns-LA, where laser interacts with plasma forming higher early excitation temperature and electron density. Herein, the results show the prominence of higher ionic states in fs plasmas despite shorter life time and cooler plasmas due to the fundamental ablation mechanisms. In fs-LA, the fast dissipation of pulse energy density into the sample with minimized thermalization incur Coulombic regime of plasma hydrodynamics where electrostatic ejection and formation of atomic, ionic, and molecular fragments is dominant. The high ionic states abundance is found to increase with laser power density ${ }^{49}$. In ns-LA, the thermalization and plasma heating results in lower ionization states ${ }^{50}$ and higher plasma parameters (both higher plasma temperature and electron density) due to the dissipation mechanism of thermal energy into sample. The contrarieties in laser energy coupling between fs and ns-LAs are clarified by craters morphologies.

Craters topology. The ablation features; i.e. mass and yield, of both $f$ s-Solstice and $n s$-Chem systems are investigated by focused ion beam scanning electron microscopy (FIB-SEM) and coherence scanning interferometry (CSI) to better evaluate the formation mechanism of coal ejecta induced by fs-LIBS system relative to that obtained by the ns-LIBS system.

FIB-SEM images. Figure 6a elucidates a precise crater with Gaussian-like profile obtained via fs-Solstice system ablation, while the crater bottom can be clearly seen. The sample surface shows no rim formed nor heat-affected zones (HAZs) of molten material. In contrast, both rim and HAZs are observed in case of $n s$-Chem ablation, see Fig. 6b. Cracks at the crater's edge are observed due to a strong fs-laser hit (a pulse train of 100 signals) in ablation with electrostatic nature which are not observed in case of $n s$-Chem ablation (single shot) and replaced with scattered HAZs of molten material formed in thermal explosion reaction. Splashed particles ejected from molten ablated mass are distinguished on coal sample after ns-LA, and according to their temperature at the landing time over the surface, they appear as HAZs or large agglomerates. For fs-LA, powdery appearance of nanoparticles spread around the crater indicates that the coal ejecta is ablated in nanoscale particle size. This is in agreement with the report by Liu et al. ${ }^{51}$ who studied the ablated aerosol vapor collected on silicon substrate after fs and ns-LAs of brass alloy. SEM images showed ejecta formed by fs-LA were $\sim 100 \mathrm{~nm}$ while those formed by ns-LA were in $100 \mathrm{~s} \mathrm{~nm}$ to $\mu \mathrm{m}$ size scale. The slow thermalization and re-solidification of the ablated matter on sample's surface are significant sources of matrix effects and fractional evaporation. It should be noted that $n s$-Chem is in optimal operation conditions of minimized HAZ to reduce matrix effects and to enhance signals repeatability. The $f$ s-Solstice system, which is not exactly working in optimal operation conditions for coal analysis, shows reduction of thermal effects and proves absence of fractional evaporation.

CSI profiles. The 3D surface profilometer images show the alteration occurred to the coal sample after laser ablation. Figure 7 shows the Gaussian beam profile of $f_{s}$-Solstice laser which yields a cone-shaped crater accompanied with absence of molten rims and HAZs. The fs-crater has a surface diameter of $617 \mu \mathrm{m}$ and depth of $350 \mu \mathrm{m}$. For $n s$-Chem system, rims as molten heights with pointed tips (look like stalactites) are formed around the crater with HAZs are shown as random thermal grooves/modifications of coal surface, see Fig. 8. The nscrater has a surface diameter of $203 \mu \mathrm{m}$ and depth of $8 \mu \mathrm{m}$. While $f s$-Solstice system employs a fs-laser with Gaussian beam profile, $n s$-Chem system has a laser source with top-hat beam profile. Therefore, assuming the ablated masses have right structures, $f s$-Solstice yielded $\sim 3.5 \mathrm{E}-05 \mathrm{~cm}^{3}$ ablation volume (considering the fs-crater has circular cone structure) and $n s$-Chem ablated $\sim 1.5 \mathrm{E}-07 \mathrm{~cm}^{3}$ (considering the ns-crater has truncated cone structure). Using the bituminous coal density $\left(1.346 \mathrm{~g} \mathrm{~cm}^{-352}\right)$, the ablated masses are $\sim 40$ and $\sim 0.2 \mu \mathrm{g}$ for $f_{s}$ - 


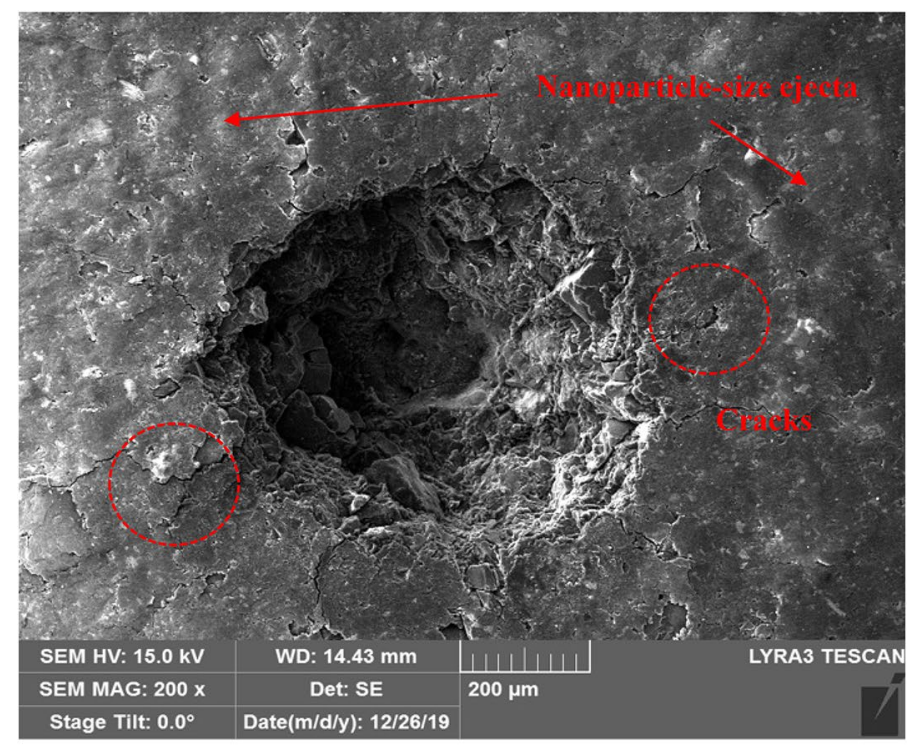

(a)

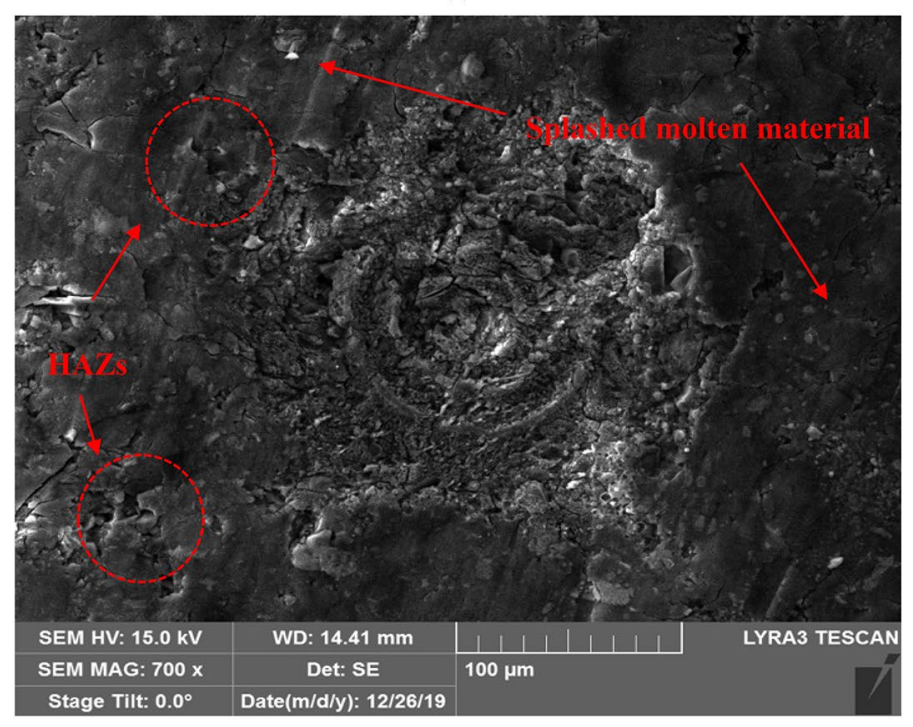

(b)

Figure 6. Focused ion beam scanning electron microscopy (FIB-SEM) images of laser ablation craters formed using (a) $f_{s}$-Solstice sytem where: no rim not heat affected zones (HAZs), cracks at the crater's edge due to strong fs laser hit, and powdery appearance of ejecta are observed; (b) $n s$-Chem system where: rim, HAZs, molten and splashed particles are observed.

Solstice and $n s$-Chem systems, respectively. The ablation yield is defined as the mass ablated per laser pulse which gives $0.4 \mu \mathrm{g} /$ pulse for fs-Solstice system (a pulse train of 100 signals) and $0.2 \mu \mathrm{g} / \mathrm{pulse}$ for $n s$-Chem system. The energy densities responsible for this ablation yield are calculated from the incident laser energies and spot sizes for each system. For $f s$-Solstice system, the energy density is: $\left(5.7 \times 100 \times 10^{-3}\right) \mathrm{J} /\left(\pi \times\left(617 \times 10^{-4}\right)^{2} \mathrm{~cm}^{2}=47.66\right.$ $\mathrm{J} \mathrm{cm}^{-2}$. For $n s$-Chem system, the energy density is: $\left(90 \times 10^{-3}\right) \mathrm{J} /\left(\pi \times\left(203 \times 10^{-4}\right)^{2} \mathrm{~cm}^{2}=69.51 \mathrm{~J} \mathrm{~cm}^{-2}\right.$. This means that $f_{s}$-Solstice system has double the ablation yield of $n s$-Chem system and ablates a mass which is 200 times more that ablated by $n s$-Chem system with $\sim 2 / 3$ of the energy density employed. Table 5 summarizes the ablation features for the evaluation of $f s$-Solstice relative to $n s$-Chem.

Several contributing factors have been discussed in literature to explain the phenomenology of enhanced energy density deposition in fs laser matter interactions, including:

1. Enhanced laser energy coupling Considerably contrastive physics of energy deposition between fs and ns-LA regimes is attributed to the pulse duration; i.e. time scales in which intense laser energy deposits. The fs laser pulse has a pulse duration $\tau_{\mathrm{L} / \mathrm{fs}}$ shorter than the electron-lattice relaxation time $\tau_{\mathrm{e}}$. Therefore, fs-pulse energy couples to electron carriers which follow Drude hydro-dynamics with various scattering and collision events to relax energy into lattice. Hot electrons cause a high-pressure buildup within the crystal which is then 


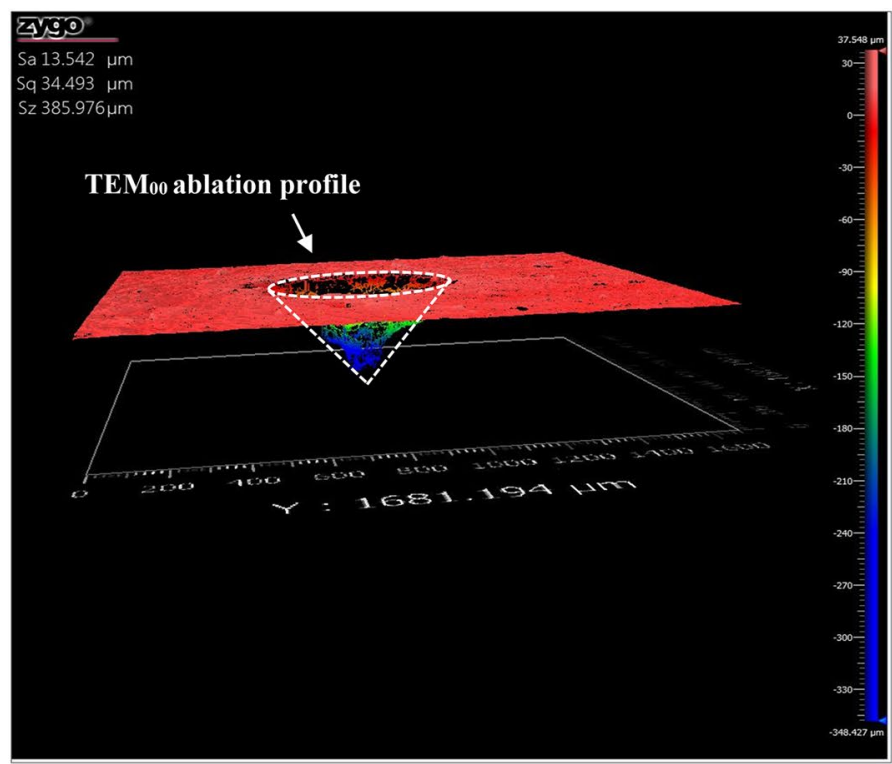

(a)

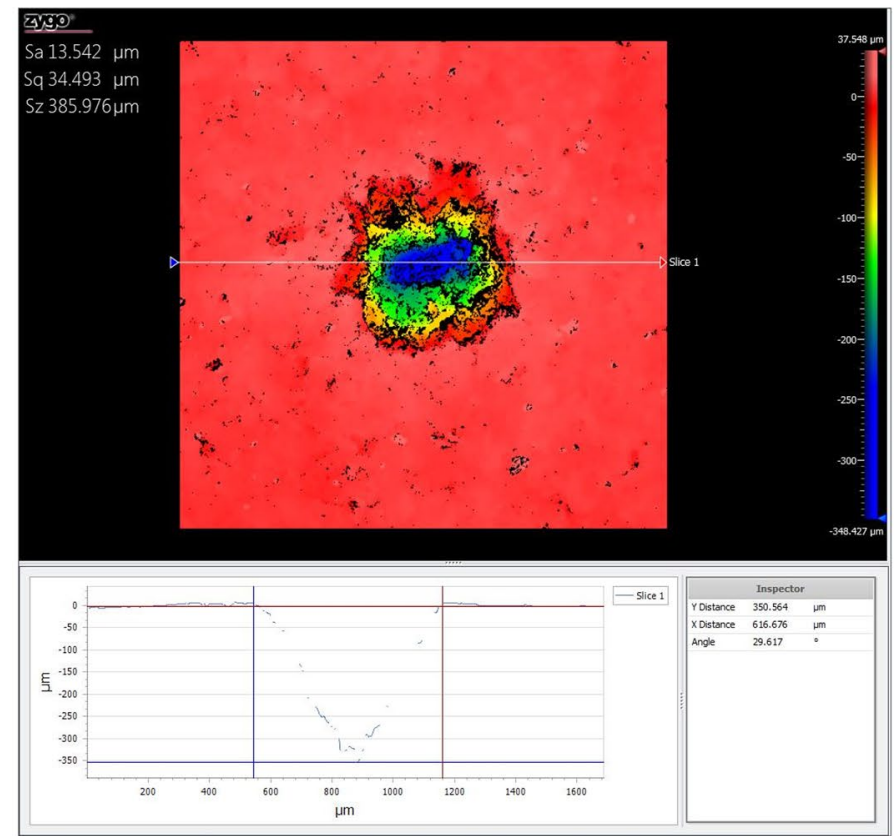

(b)

Figure 7. 3D surface profilometer images of coal sample after ablation by $f$ s-Solstice system: (a) is the $3 \mathrm{D}$ profile and $(\mathbf{b})$ is surface profile view. The ablated volume has a circular-cone structure accompanied with absence of molten rims and heat affected zones. The crater diameter is $617 \mu \mathrm{m}$ and its depth is $350 \mu \mathrm{m}$.

released via mechanical expansion in electrostatic ablation. The extremely rapid isochoric heating of lattice pushes the system into metastable state where homogenous nucleation of gas bubbles in solid-vapor phase explosion takes place. Moreover, mechanical fragmentation and spinodal decomposition are two effective ablation mechanisms happening at high energy density regime. The two-temperature model $^{53,54}$ is used to describe the energy relaxation between electrons and lattice, as:

$$
\begin{gathered}
C_{e} \frac{\partial T_{e}}{\partial t}=-\frac{\partial\left[-k_{e} \frac{\partial T_{e}}{\partial z}\right]}{\partial z}-\gamma\left(T_{e}-T_{i}\right)+I_{a} \alpha \exp (-\alpha \mathrm{z}) \\
C_{i} \frac{\partial T_{i}}{\partial t}=\gamma\left(T_{e}-T_{i}\right)
\end{gathered}
$$




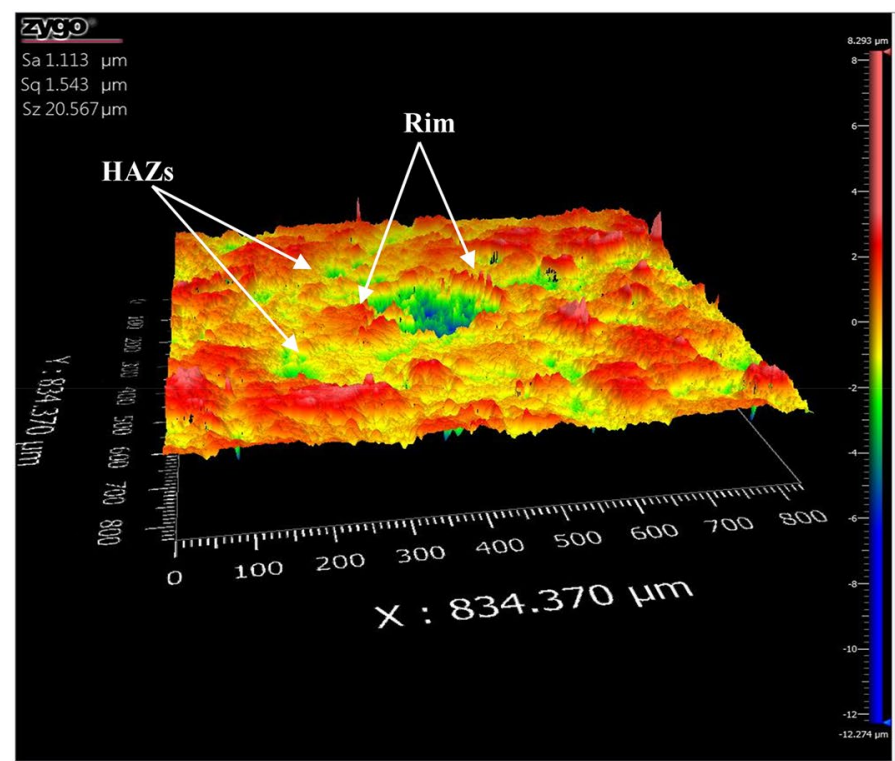

(a)
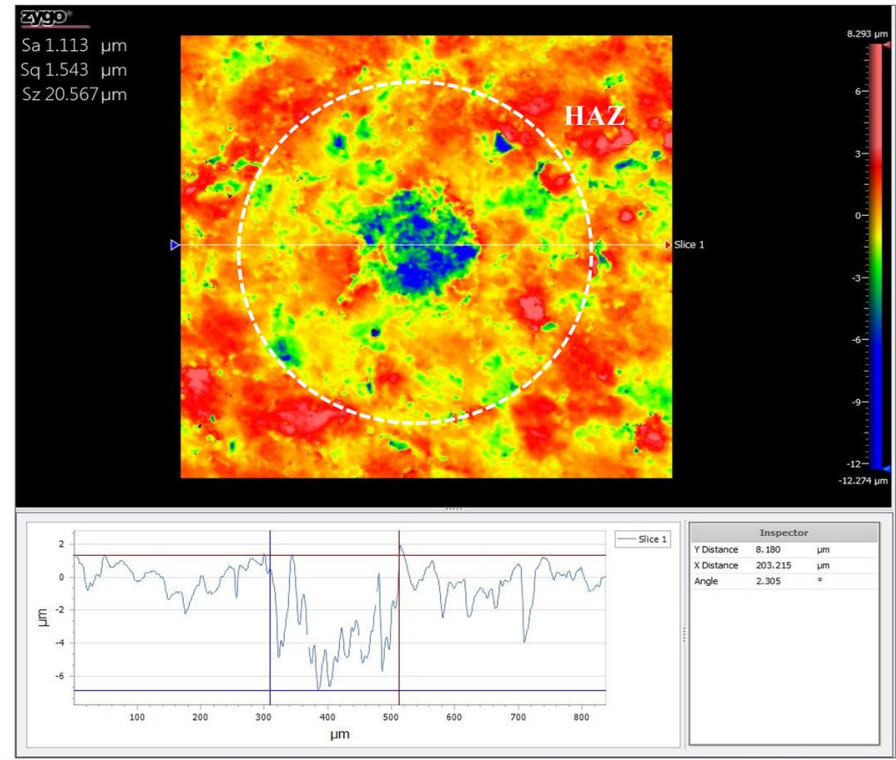

(b)

Figure 8. $3 \mathrm{D}$ surface profilometer of coal sample after ablation by $n s$-Chem system: (a) is the $3 \mathrm{D}$ profile and (b) is surface profile view. The ablated volume can be simulated as a truncate-cone structure with clear thermal effects; rims and heat affected zones (HAZs). The crater diameter is $203 \mu \mathrm{m}$ and its depth is $8 \mu \mathrm{m}$.

\begin{tabular}{|l|l|l|}
\hline & fs-Solstice & ns-Chem \\
\hline Crater width $/ \mu \mathrm{m}$ & 617 & 203 \\
\hline Crater depth/ $\mu \mathrm{m}$ & 350 & 8 \\
\hline Crater volume $/ \mathrm{cm}^{3}$ & $3.5 \times 10^{-5}$ & $1.5 \times 10^{-7}$ \\
\hline Mass of ejecta/ $\mu \mathrm{g}$ & 40 & 0.2 \\
\hline Ablation yield/ $\mu \mathrm{g}$ per pulse & 0.4 & 0.2 \\
\hline Energy density/ $\mathrm{cm}^{-2}$ & 47.66 & 69.51 \\
\hline
\end{tabular}

Table 5. Craters and ablation features of $f s$-Solstice and $n s$-Chem systems for coal samples analysis. 


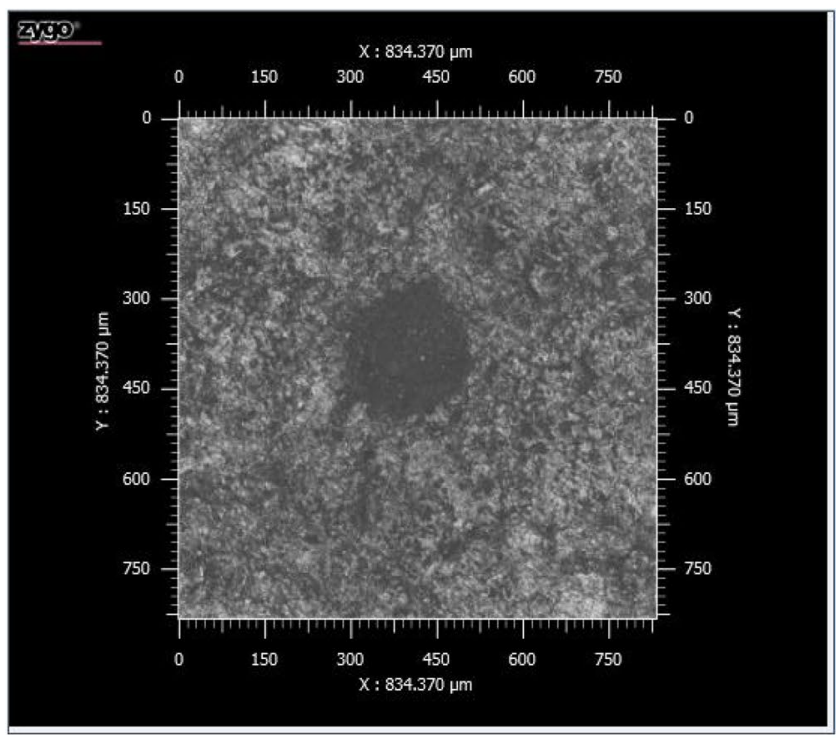

(a)

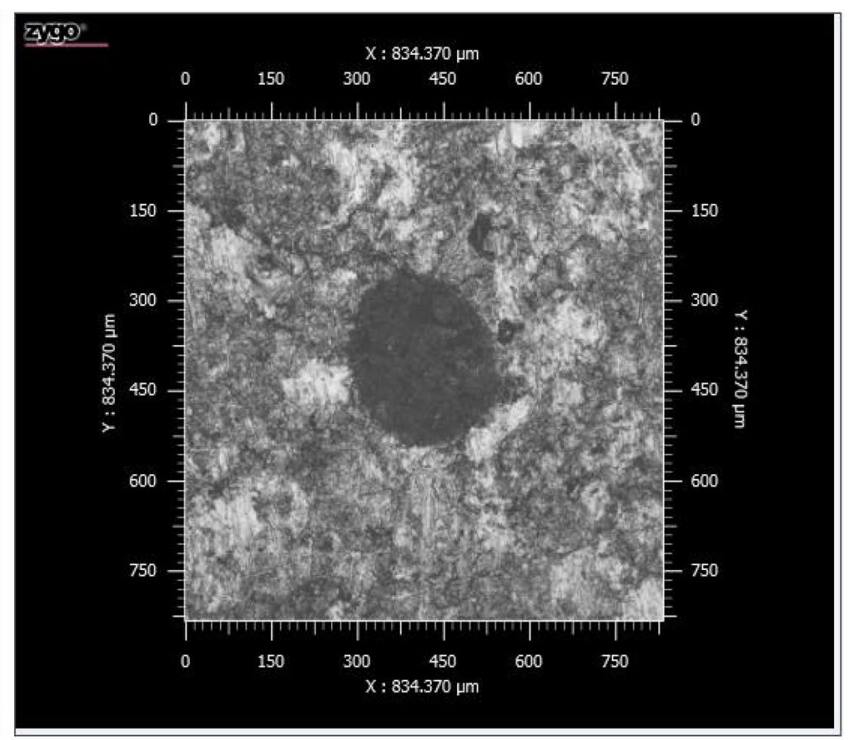

(b)

Figure 9. 2D surface profilometry images of single shots ablation using fs-Solstice system in (a) and $n s$-Chem system in (b) clarifying the reduced thermal effects of fs-LA.

where; $C_{e}$ is the heat capacity per unit volume of the electron subsystem, $T_{e}$ is electron temperature, $Q(z)$ is the heat flux which is a function at $\mathrm{z}$ direction perpendicular to the target surface and given by: $Q(z)=-k_{e}\left(\frac{\partial T_{e}}{\partial z}\right), \gamma$ is the electron-lattice coupling parameter, $T_{i}$ is lattice temperature, $S$ is the laser heating source function and given by: $S=A \alpha I(t) \exp -\alpha \mathrm{z}, C_{i}$ is the heat capacity per unit volume of the lattice subsystem, $k_{e}$ is the electron thermal conductivity, $A=1-R$ is the surface transmittivity, $\alpha$ is the material absorption coefficient, and $I_{a}$ is the absorbed laser intensity. For a fs-pulse, $\tau_{\mathrm{L} / \mathrm{fs}}<<\tau_{\mathrm{e}}$, and hence, $\left.\left(C_{e} T_{e}\right) / \mathrm{L} / \mathrm{fs}\right) \gg \gamma T_{e}$ and $\mathrm{D}_{\mathrm{e}} \tau_{\mathrm{L} / \mathrm{fs}}<\alpha^{-2}$ where $\mathrm{D}_{\mathrm{e}}$ is the electron thermal diffusivity. The threshold fluence for fs-evaporation is given by: $F_{t h / f s} \approx \rho \Omega\left(D_{e L / f s}\right)^{1 / 2}$. If the electron heat conduction is neglected, then the fsplasma expansion is adiabatic, ultrafast, and non-thermal ablation where threshold fluence is minimized with pulse width. Figure 9 shows 2D CSI profiles of a fs-single shot (not a pulse train) and a ns-single shot of a coal sample. The reduced thermal effect and enhanced laser energy deposition are clearly seen for $f s$ Solstice pulse relative to $n s$-Chem one.

2. Enhanced residual thermal energy deposition At high fluences and prior to the hydrodynamic expansion of ablated ejecta, direct ionization of ambient gas (collisional environment) can occur due to highly energetic electron-carriers with fs-pulse energy directly deposited into them. A high-pressure plasma layer is generated at the interaction zone which is believed ${ }^{55-57}$ to enhance electrons-lattice thermal coupling by retaining (or confining) coupling process at a time interval required for rarefaction wave (decreased density and pressure, molecules stretch time) to travel from plasma periphery to center of irradiated spot and then enhance the re-deposition of ablated ejecta-see Fig. 10.

3. Reduced threshold fluence of ablation in $f$ s-train pulse Incubation phenomena ${ }^{58,59}$ is behind the reduced threshold fluence of ablation in a fs-train pulse. Physical ansatzes are developed to link the incubation to the formation of laser-induced states formed due to the storage cycle of thermal stress-strain energy (material fatigue) and the creation of laser induced defects, ripples, and nano-cracking ${ }^{60-62}$. Meanwhile, surface reflectivity was observed to decrease in fs train pulse ${ }^{63,64}$. The Nth declined fluence is reported as: $F_{t h / f_{s}}^{N} \approx F_{t h / f_{s}}^{1} N^{-\xi}$ where $F_{t h / f s}^{1}$ is the single-pulse threshold fluence and $\xi$ is the incubation coefficient. For a beam with a Gaussian beam profile, the diameter of the ablated crater is related to applied fluence ${ }^{65}: D^{2}=2 \omega_{o}^{2} \ln \left(\mathrm{F} / \mathrm{F}_{\mathrm{th}}\right)$; where $\omega_{o}$ is $1 / \mathrm{e}^{2}$ radius of laser light distribution and $D$ is the formed crater diameter. The threshold fluence at the 100th pulse in the train pulse is then deduced: $F_{t h / f s}^{100} \sim 0.102 \mathrm{~J} \mathrm{~cm}^{-2}\left(D=617 \mu \mathrm{m}\right.$ and $\left.\omega_{o}=176 \mu \mathrm{m}\right)$.

Plasma parameters. Spectral lines characteristics (intensities, resolution, widths, and repeatability) and crater morphologies (depth, width, and precision) are causally connected to the matter physicochemical structure and laser-matter interaction properties; i.e. plasma parameters. Hence, plasma parameters (plasma temperature and electron number density) are utilized as an evaluation measure to see the parametric plasma conditions led to the corresponding spectral features, abundant species detected in plasmas, and craters topology by $f s$-Solstice system relative to those generated by $n s$-Chem one. Plasma temperatures are extrapolated from the electronic temperature of plasma components assuming a thin plasma in local thermodynamic equilibrium according to Griem's criterion ${ }^{66}$. Boltzman's law $^{67}$ is applied for four closely-spaced Mg II lines to deduce plasma temperature as: 


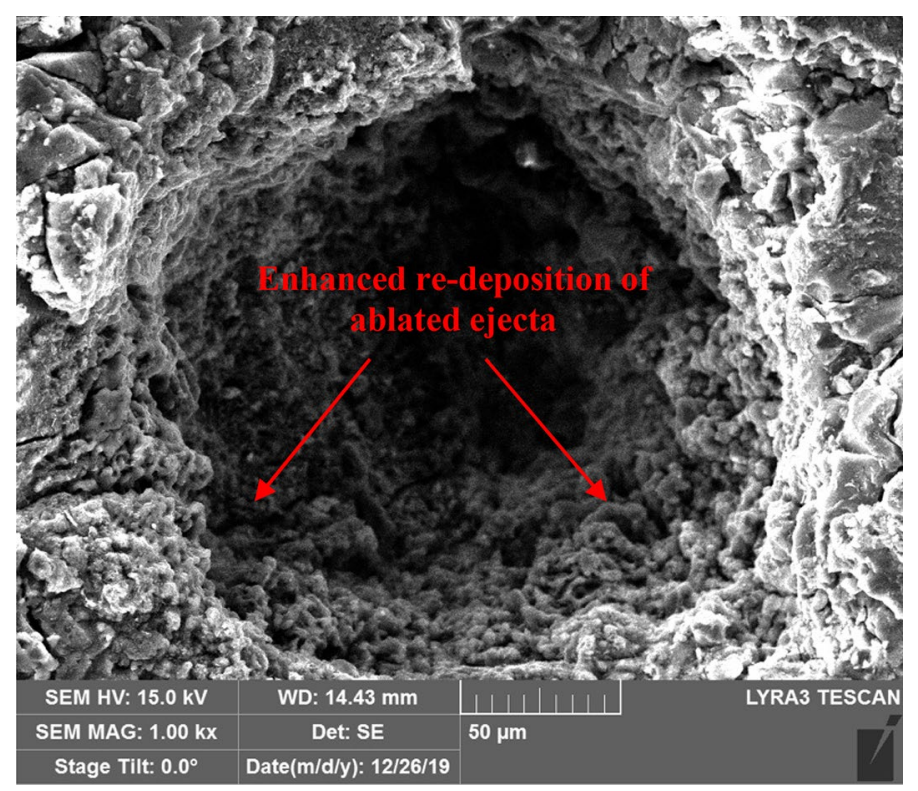

Figure 10. Focused ion beam scanning electron microscopy (FIB-SEM) image of the interior part of coal crater ablated by $f s$-Solstice system (a train pulse of 100 signals). The re-deposited ablated ejecta inside the crater resemble those formed on the surface as nanoparticle-sized which confirms the enhanced thermal energy deposition for fs-LA.

\begin{tabular}{|l|l|l|l|l|l|}
\hline$\lambda / \mathbf{n m}$ & $\mathbf{E}_{\mathbf{k}} / \mathbf{e V}$ & $\mathbf{E}_{\mathbf{i}} / \mathbf{e V}$ & $\mathbf{A}_{\mathbf{k i}} / \mathbf{s}^{-1}$ & $\mathbf{g}_{\mathbf{k}}$ & $\mathbf{g}_{\mathbf{i}}$ \\
\hline 279.10 & 8.86 & 4.42 & $1.63 \times 10^{-3}$ & 6 & 2 \\
\hline 279.56 & 4.43 & 0.00 & $2.60 \times 10^{8}$ & 2 & 4 \\
\hline 279.81 & 8.86 & 4.43 & $4.79 \times 10^{8}$ & 4 & 6 \\
\hline 280.27 & 4.42 & 0.00 & $2.57 \times 10^{8}$ & 2 & 2 \\
\hline
\end{tabular}

Table 6. List of Mg II lines for plasma temperature calculation and corresponding spectroscopic parameters.

$$
\ln \left(\frac{\lambda_{\mathrm{ki}} \mathrm{I}_{\mathrm{ki}}}{\mathrm{g}_{\mathrm{k}} \mathrm{A}_{\mathrm{ki}}}\right)=-\left(\frac{\mathrm{E}_{\mathrm{k}}}{\mathrm{k}_{\mathrm{B}} \mathrm{T}}\right)+\mathrm{C}
$$

where; $\lambda_{k i}, I_{k i}, g_{k}, A_{k i}, E_{k}, k_{B}$, and $C$ are emission wavelength, integral intensity of emission line, degeneracy of upper level energy, transition probability, upper-level energy, Boltzmann constant, and a constant, respectively. Plasma temperatures are obtained using slope of the fitted line. Non-resonant ionic Mg II lines: 279.10, 279.56, 279.81 , and $280.27 \mathrm{~nm}$ are selected to deduce the temperature. Our choice is based on using lines with same ionization states and closely-spaced to reduce the effect of instrumental efficiency bias. For each sample, 100 spectra are averaged and normalized to the whole spectral area then $\mathrm{Mg}$ II lines are used to deduce the sample's plasma temperature. The Mg II lines exhibit $\sim 20$ times higher normalized peak intensities in case of $f$ s-Solstice spectra relative to $n s$-Chem spectra. This is attributed to the accumulation over fs train pulses, higher resolution, and detector sensitivity of the spectrometer used in the fs system. Nevertheless, the integrated line intensities of $\mathrm{Mg}$ II lines are used without background subtraction to encounter for population-averages of the local electronic temperature through the lifelong detection window for each system. Table 6 collects the spectral line parameters from NIST database ${ }^{20}$.

The electron density is determined by using the full-width at half maximum (FWHM) of spectral lines according to Stark broadening ${ }^{68}$ :

$$
N_{e}=\left[\Delta \lambda_{\text {Stark }} \times \frac{10^{16}}{2 \omega}\right]
$$

where; $\omega$ is electron impact parameter, and $\Delta \lambda_{\text {Stark }}$ is FWHM of Stark broadened profile. Lines' profiles are affected by different broadening mechanisms. Stark broadening and instrumental broadening are accounted for in laser-induced plasmas; considering contribution of other broadening mechanisms is ignorable. The C I transition at $247.86 \mathrm{~nm}$ is used for electron density measurements and is fitted using a Voigt profile which considers Stark broadening by Lorentzian profile and instrumental broadening by Gaussian profile. Stark broadening is 


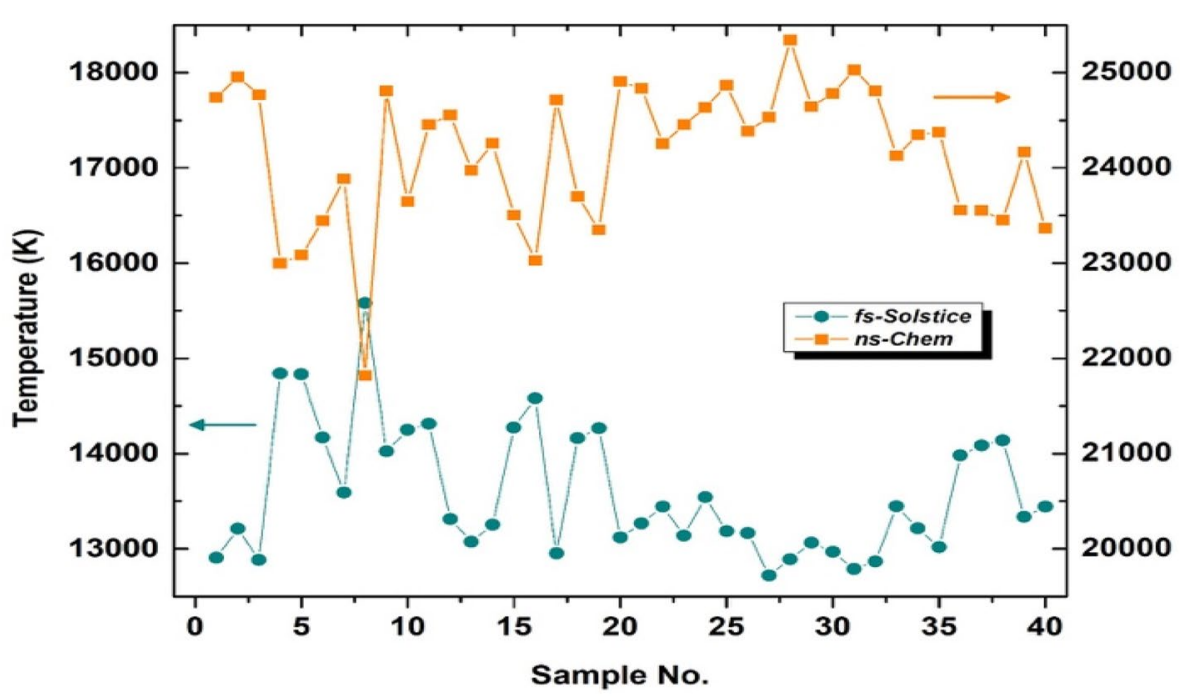

(a)

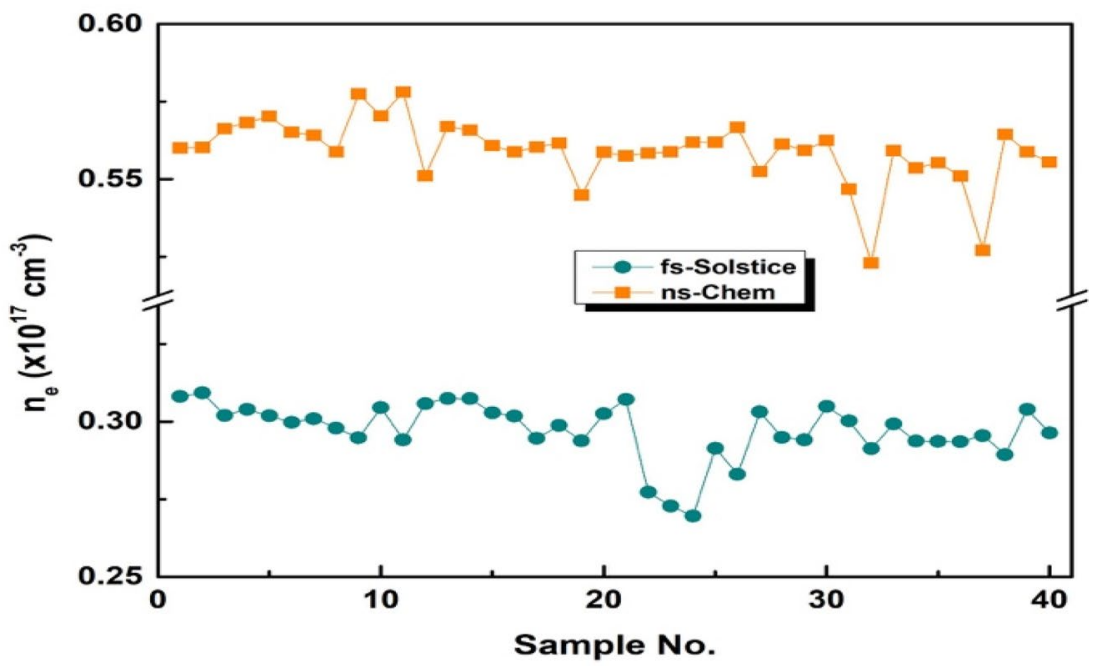

(b)

Figure 11. Plasma temperatures in (a) and electron densities in (b) for 40 coal samples ablated with $f_{s}$-Solstice and $n s$-Chem plasmas. The average temperature value for fs system is $13,583 \pm 679 \mathrm{~K}$ while for $\mathrm{ns}$ system is $24,151 \pm 732 \mathrm{~K}$. The average electron number densities are $2.97 \pm 0.09 \times 10^{16} \mathrm{~cm}^{-3}$ for fs plasma and $5.59 \pm 0.10 \times 10^{16} \mathrm{~cm}^{-3}$ for ns plasma.

de-convoluted after inserting instrumental broadening for each system (22 pm and $0.5 \mathrm{~nm}$ FWHM for $f$ s-Solstice and $n s$-Chem systems respectively measured using a low-pressure mercury lamp) as follows:

$$
\Delta \lambda_{\text {measured }(\text { Voigt })}=\left[\left(\frac{\Delta \lambda_{\text {Stark }(\text { Lorentz })}}{2}\right)^{2}+\left(\Delta \lambda_{\text {Instru. (Gauss })}\right)^{2}\right]^{\frac{1}{2}}+\left(\frac{\Delta \lambda_{\text {Stark }(\text { Lorentz })}}{2}\right)
$$

Figure 11 depicts the plasma temperatures (in a) and electron densities (in b) for 40 coal samples analyzed by each system. The average plasma temperature of all samples analyzed by $f$ s-Solstice system is $13,583 \pm 679 \mathrm{~K}$. While, it is $24,151 \pm 732 \mathrm{~K}$ for $n s$-Chem system. Similarly, the average electron density is $2.97 \pm 0.09 \times 10^{16} \mathrm{~cm}^{-3}$ for $f s$-Solstice and is $5.59 \pm 0.10 \times 10^{16} \mathrm{~cm}^{-3}$ for $n s$-Chem. FWHM of other minor elemental lines (not shown) expressed alike trend suggesting denser plasmas obtained by $n s$-Chem system ablation parameters. One might consider that hotter and denser plasma conditions are better to obtain more ablation ejecta and spectral information. While, $f s$-Solstice evaluation results of the performance measures: RSFs and craters topology with plasma parameters show that cooler and less dense plasma may form less chaotic conditions for different elemental species to evolve and gentle craters to form. The laser-plasma interaction is virtually absent, and thereby, fsplasmas are overcoming the shielding and re-heating effects typically caused by ns-laser excitation ${ }^{33}$. Therefore, $f_{s}$-Solstice plasmas have lower plasma temperatures and less electron density values relative to $n s$-Chem plasmas. From physics point of view, as soon as the formed plasma temperature in the interaction zone reaches energy 


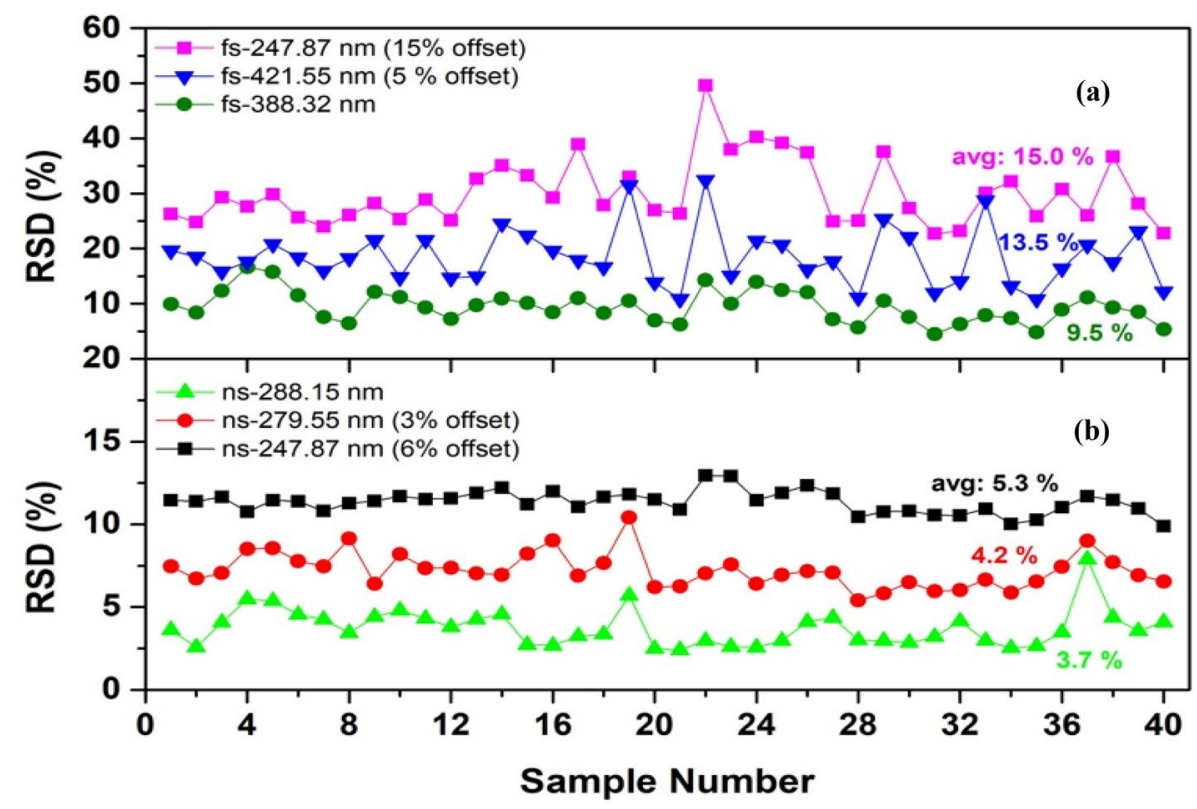

Figure 12. Relative standard deviations (RSDs) of 100 spectra for the 40 coal samples ablated by: (a) fs-Solstice, and (b) $n$-Chem systems with average RSDs of $9.7 \%$ and $2.5 \%$ for the two systems respectively. Offsets are to avoid points' overlapping for clarity.

magnitudes enough for breaking/melting, vaporization, and ionization of matter species, it validates for spectroscopy elemental detection. In a sense, an ideal plasma performs stoichiometric ablation with least chaotic behavior. The electrostatic ablation nature and the absence of laser-plasma interaction promote $f s$-Solstice plasma as competitive source for non-stochastic plasma parameters.

Measurement-to-measurement repeatability. Spectral repeatability is accessed by average measurement-to-measurement RSDs of raw spectral lines. Figure 12 shows RSDs of 100 measurements (ablation matrix of $10 \times 10$ positions) for 40 coal samples ablated by $f$ s-Solstice (in a) and $n s$-Chem (in b) systems. The average RSD of C I $247.87 \mathrm{~nm}$ line for all samples is $15 \%$ for $f s$-Solstice spectra; 3 times higher than that obtained by $n s$-Chem spectra (average RSD of 5.3\%). The Sr II $421.55 \mathrm{~nm}$ line and the molecular band CN at $388.32 \mathrm{~nm}$ show average RSDs of $13.5 \%$ and $9.5 \%$ respectively for $f s$-Solstice spectra. While, other lines including Si I line at $288.15 \mathrm{~nm}$ and $\mathrm{Mg}$ II at $279.55 \mathrm{~nm}$ show lower average RSDs of $3.7 \%$ and $4.2 \%$ respectively in case of $n s$-Chem spectra. To get an overall estimation of each system's repeatability, the 100 lines with highest repeatability obtained by each system are investigated. For $f s$-Solstice system, 100-highest repeatability lines have average RSD of $9.7 \%$. The repeatable lines are branches of $\mathrm{CN}$ or $\mathrm{C}_{2}$ bands due the stable formation of molecules; i.e. fragmentation. For $n s$-Chem spectra, 100-highest repeatability lines have average RSD of $2.5 \%$. The repeatable lines are atomic/ionic lines of mineral elements.

The fact that $f s$-Solstice system has: stable crater morphology, ionized plasma cloud which is not interacting with the laser tail, and ejecta formed in a cool plasma with considerably low chaotic behavior in a short lifetime, encounter the expectation of higher signals repeatability relative to $n s$-Chem system. However, $n s$-Chem system is optimized for best-signals repeatability and precise quantitative analysis as an offline coal analyzer already operating in power plants. The work on the direction of optimizing the $f$ s-Solstice system repeatability for coal analysis would uncover interesting aspects about plasma modeling based on physical background where accuracy is significantly boosted.

Industrial scale-up of fs-LIBS: applicability and problems. Ultrafast lasers are unique by incredibly high peak intensities and interaction timescale faster than the lattice disorder and heat diffusion. Since their development over half a century, researchers continue to uncover new phenomena about laser-matter interactions. As for spectroscopic techniques, advances provided by femtosecond lasers have been directed to solve different analytical tasks. LIBS benefits from femtosecond lasers and the review paper by Labutin et al.$^{69}$ foresaw a brighter future for numerous fs-LIBS applications due to state-of-the-art findings reviewed in studies. Howbeit, a slow mainstream adoption of fs- LIBS is observed relative to the analytical and industrial problems the technique is capable to solve. This is attributed to: (1) Unavailability of reliable/stable high energy femtosecond lasers; (2) Lack of fs-physics understanding quadrated with analytical capabilities; (3) Commercial restraints such as high cost, maintenance, and performance; (4) Presence of other affordable techniques. In fact, the first review paper of fs-LIBS by Gurevich et al. ${ }^{70}$ outlined two important points about scaling up fs-LIBS technique. The first point is that a replacement of current widely-used technique cannot be expected without a better performance in terms of analytical figures-of-merit by fs-LIBS based on understood plasma formation mechanisms. 


\begin{tabular}{|c|c|c|c|}
\hline Performance measure & fs-Solstice & ns-Chem & Evaluation \\
\hline Correlation coefficients & $\begin{array}{l}r \geq 0.949 \\
\text { Different lines with most identified within } \\
\text { molecular bands }\end{array}$ & $\begin{array}{l}r \leq 0.923 \\
\text { Same lines correlated to carbon content and heat } \\
\text { value }\end{array}$ & $\begin{array}{l}\text { Higher representativeness and selectiveness of } \\
f s \text {-Solstice spectra to coal property } \\
\text { Formation of molecular fragments correlated to } \\
\text { parent molecules due to fs-laser ablation nature }\end{array}$ \\
\hline Relative sensitivity factor & $\mathrm{RSFs} \approx 2-10$ & & $\begin{array}{l}\text { Enhanced detection sensitivity of ionic and } \\
\text { atomic species by } f \text { s-Solstice system }\end{array}$ \\
\hline Craters topology & $\begin{array}{l}\text { No rim nor heat-affected zone } \\
\text { Ejecta in nanoparticle-size } \\
\text { Ablated mass }=\sim 40 \mu \mathrm{g} \text { by } 47.66 \mathrm{~J} \mathrm{~cm}^{-2}\end{array}$ & $\begin{array}{l}\text { Clear rim and heat-affected zone } \\
\text { Splashed and molten material } \\
\text { Ablated mass }=\sim 0.2 \mu \mathrm{g} \text { by } 69.51 \mathrm{~J} \mathrm{~cm}^{-2}\end{array}$ & $\begin{array}{l}\text { Higher ablation yield of } f_{s} \text {-Solstice system }(0.4 \mu \mathrm{g} / \\
\text { pulse relative to } 0.2 \mu \mathrm{g} / \text { pulse for } n s \text {-Chem system) } \\
\text { due to enhanced laser-energy coupling }\end{array}$ \\
\hline Plasma parameters & $\begin{array}{l}\text { Plasma temperature }=13,583 \pm 679 \mathrm{~K} \\
\text { Electron density }=2.97 \pm 0.09 \times 10^{16} \mathrm{~cm}^{-3}\end{array}$ & $\begin{array}{l}\text { Plasma temperature }=24,151 \pm 732 \mathrm{~K} \\
\text { Electron density }=5.59 \pm 0.10 \times 10^{16} \mathrm{~cm}^{-3}\end{array}$ & $\begin{array}{l}\text { Cooler and less-dense fs-plasma incorporate less } \\
\text { chaotic behavior for stable ablation }\end{array}$ \\
\hline Repeatability & RSDs $=9.5-15 \%$ & $\mathrm{RSDs}=3.7-5.3 \%$ & $\begin{array}{l}\text { Optimization of } f \text { s-Solstice system repeatability } \\
\text { is needed }\end{array}$ \\
\hline
\end{tabular}

Table 7. Performance measures and evaluation results of $f s$-Solstice system relative to $n s$-Chem for coal analysis.

The second point is that a booming growth of ongoing trends such as femtosecond micromachining and surgery applications can provide a way for simplified, compact, and all-solid-state femtosecond lasers. This means that: (1) Ablation physics plays a significant rule in understanding the analytical capabilities and limitations of fsLIBS. Without such detailed physics understanding, effective optimization of fs-LIBS instrumentation is hardly possible; (2) Industrial applications of fs-LIBS and trendy applications (e.g. thin films deposition, quantum dots synthesis, chemical imaging, standoff environmental sensing, etc.) where fs-LIBS performs inline operation monitoring are gateways for effective commercialization where cost and size reduce significantly. At present, some research groups around the world are focusing on both directions of analytical performance of fs-LIBS quadrated to its ablation physics and industrially-related applications. We believe that for coal analysis industry with a huge market size, operation efficiency and accuracy have decisive impacts on a technique's utilization and fs-LIBS introductory-study presented in this letter is a challenging new task for a wide research direction of in-field adaption of the technique. Table 7 summarizes the performance measures and evaluation results of $f_{s}$-Solstice system under assessment relative to $n s$-Chem system.

\section{Conclusion}

Femtosecond lasers provide enhanced analytical capabilities for different LIBS applications. In this work, we have conducted an evaluation study of a one-box femtosecond laser system for the application of coal property analysis relative to an industrially-applied coal analyzer. The evaluation study is based on using 5 different objectives/measures: spectral correlations, relative sensitivity factors, craters topology, plasma parameters, and repeatability - which cover different analytical performance aspects. The fs-LIBS system reveals competitive results to those obtained by the reference coal analyzer:

- Higher representativeness (correlation coefficients) of fs-spectra to coal properties with different lines correlated to each coal property (higher selectiveness).

- Enhanced detection sensitivity and presence/abundance of ionic lines in fs-plasmas with relative sensitivity factors of 2-10.

- Precise ablation craters with no rim nor heat-affected zones of splashed/molten material due to enhanced laser energy coupling for fs-plasmas. The fs-system has higher ablation yield of $0.4 \mu \mathrm{g} / \mathrm{pulse} \mathrm{vs.} 0.2 \mu \mathrm{g} / \mathrm{pulse}$ for the reference system.

- Cooler and less-dense fs-plasmas incorporate less chaotic behavior for stable ablation.

- Further studies have to be conducted for in-field optimization of fs-system, includes: signals repeatability for precise quantitative results, operation under harsh conditions, customization for different installation sets in power plants, etc.- - the reference coal analyzer has higher signals' repeatability.

\section{Materials and systems}

Samples. Samples used in this study were 40 standard powdery bituminous coal samples. Table 8 shows the certified chemical analysis of $\mathrm{C}$, heat value, volatile, and ash contents. Air-dried coal pellets were pressed using a hydraulic tablet machine at 25 -ton pressure for $3 \mathrm{~min}$. Each pellet contained $\sim 3 \mathrm{~g}$ powder coal and formed in dimensions of $30 \mathrm{~mm}$ diameter and $3 \mathrm{~mm}$ thickness after pressing. Two sets of samples were prepared under the same conditions, each to be analyzed by a system.

Coal-analysis systems. Femtosecond-LA system (fs-Solstice). A one-box ultrafast Ti:Sapphire amplifier (SolsticeAce, Spectra Physics, USA) was targeted for a performance test as a coal analysis system (Fig. 13a). Inside the amplifier box (with $125 \times 68 \times 29 \mathrm{~cm}$ dimensions) are four independent modules: regenerative amplifier, stretcher/compressor, seed laser, and pump laser. Two power supplies and chillers are connected to the system for seed (Mai Tai) and pump (Empower) lasers operation. The system produces laser pulses centered at $800 \mathrm{~nm}$ with linear polarization and it is user configurable to operate over: $2-7 \mathrm{~mJ}$ pulse energy, 35-120 fs pulse width, and $4-1000 \mathrm{~Hz}$ repetition rate. The system employs adjustment-free EternAlign internal optical mounts to maximize long-term stability and operation (Energy stability of $<0.5 \%$ rms over $24 \mathrm{~h}$ operation). 


\begin{tabular}{|c|c|c|c|c|c|}
\hline Sample code & Sample no. & Carbon (wt\%) & Heat value $\left(\mathrm{MJ} \mathrm{kg}^{-1}\right)$ & Volatile (wt\%) & Ash (wt\%) \\
\hline ZBM096 & $\# 1$ & 81.45 & 31.86 & 11 & 10.09 \\
\hline ZBM098A & $\# 2$ & 78.58 & 32.5 & 31.68 & 8.25 \\
\hline ZBM099 & $\# 3$ & 79.6 & 31.4 & 15.3 & 10.41 \\
\hline ZBM100A & $\# 4$ & 49.3 & 19.42 & 11.7 & 41.22 \\
\hline ZBM100B & $\# 5$ & 46.46 & 18.16 & 11.81 & 43.85 \\
\hline ZBM100C & $\# 6$ & 53.69 & 20.98 & 13.64 & 34.14 \\
\hline ZBM101 & $\# 7$ & 67.08 & 26.85 & 18.5 & 22.8 \\
\hline ZBM102 & $\# 8$ & 57.82 & 22.45 & 30.43 & 25.88 \\
\hline ZBM103A & $\# 9$ & 56.15 & 21.64 & 13.77 & 29.66 \\
\hline ZBM104 & $\# 10$ & 53.63 & 21.33 & 13.5 & 33.56 \\
\hline ZBM105 & $\# 11$ & 53.16 & 20.65 & 14 & 31.8 \\
\hline ZBM106 & $\# 12$ & 72.45 & 29.44 & 30.31 & 13.68 \\
\hline ZBM108 & $\# 13$ & 79.02 & 30.56 & 11.3 & 11.98 \\
\hline ZBM108A & $\# 14$ & 78.45 & 30.45 & 11.72 & 12.21 \\
\hline ZBM111A & $\# 15$ & 74.16 & 29.72 & 33.64 & 9.62 \\
\hline ZBM111B & $\# 16$ & 72.05 & 28.85 & 33.71 & 11.74 \\
\hline ZBM111C & $\# 17$ & 77.14 & 31.25 & 31.29 & 8 \\
\hline ZBM112 & $\# 18$ & 59.65 & 23.72 & 28.5 & 25.17 \\
\hline ZBM112A & $\# 19$ & 61.2 & 24.35 & 28.96 & 23.5 \\
\hline ZBM113 & $\# 20$ & 78.5 & 32.3 & 33.7 & 8.08 \\
\hline ZBM113A & $\# 21$ & 76.5 & 31.73 & 32.77 & 9.54 \\
\hline ZBM114 & $\# 22$ & 74.6 & 29.5 & 33.3 & 6.97 \\
\hline ZBM115 & $\# 23$ & 76.36 & 30.18 & 33.2 & 4.66 \\
\hline ZBM120 & $\# 24$ & 77.28 & 30.57 & 31.74 & 6.38 \\
\hline ZBM121 & $\# 25$ & 78.15 & 31.18 & 31.8 & 6.08 \\
\hline ZBM121A & $\# 26$ & 75.34 & 29.93 & 23.77 & 11.06 \\
\hline GBW11101G & $\# 27$ & 79.02 & 31.99 & 19.43 & 10.94 \\
\hline GBW11102X & $\# 28$ & 67.12 & 27.12 & 30.1 & 16.47 \\
\hline GBW11102Y & $\# 29$ & 69.64 & 28.4 & 33.32 & 13.61 \\
\hline GBW11107C & $\# 30$ & 78.16 & 31.89 & 28.42 & 8.88 \\
\hline GBW11107D & $\# 31$ & 75.12 & 30.57 & 33.07 & 9.01 \\
\hline GBW11108P & $\# 32$ & 66.92 & 27.01 & 30.01 & 16.26 \\
\hline GBW11108Q & $\# 33$ & 68.29 & 28.03 & 32.5 & 15.88 \\
\hline GBW11109N & $\# 34$ & 65.59 & 27.06 & 35.07 & 17.06 \\
\hline GBW11109O & $\# 35$ & 66.04 & 27.23 & 35.17 & 16.6 \\
\hline GBW11109Q & $\# 36$ & 53.66 & 22.03 & 29.28 & 31.01 \\
\hline GBW11110M & $\# 37$ & 44.99 & 17.84 & 17.13 & 42.14 \\
\hline GBW11110Q & $\# 38$ & 57.01 & 22.97 & 20.39 & 28.98 \\
\hline GBW11111O & $\# 39$ & 68.59 & 27.98 & 26.81 & 18.5 \\
\hline GBW11111P & $\# 40$ & 59.33 & 23.84 & 24.82 & 26.86 \\
\hline
\end{tabular}

Table 8. Certified chemical composition of coal samples utilized in this study.

The temperature control unit (TCU) monitors a safe and constant temperature and humidity environment for the Ti:Sapphire crystal. The timing and delay generator (TDG) synchronizes the action of Pockels cells, seed laser, and pump lasers, and provides output signals which allow external instruments to capture laser-firing signals. The output laser beam is a $\mathrm{TEM}_{00}$ Gaussian beam profile with beam quality $\mathrm{M}^{2}$ of $<1.25$ and a diameter of $\sim 10 \mathrm{~mm}$. In the studied configuration, TDG was used to externally trigger a laboratory delay generator (DG645, Stanford Research Systems, USA) which enabled precise delays to the laser pulse. The laser beam was first reflected by three 730-820 nm ultrafast mirrors, then focused onto sample surface by a plano-convex lens (Fused silica, $\mathrm{f}=200.23 \mathrm{~mm}, \mathrm{~d}=25.4 \mathrm{~mm}$, and $1.6 \mathrm{~mm}$ central thickness). The mirrors and focusing lens graphed group delay dispersion of $\sim 0$ and $36 \mathrm{fs}^{2}$ for wavelengths centered at $800 \mathrm{~nm}$, respectively. An auto-correlator and a power-meter were used to measure the pulse width and energy drifts before and after the experiment. A sample holder was positioned above a computerized XY translation stage (PT1-Z8, Thorlabs, USA). Plasma emission was collected using fused silica collimating lenses (UV98 condenser, LTB, Germany) set at $45^{\circ}$ observation angle and connected to an optical fiber. An ICCD echelle-spectrometer (Aryelle-Butterfly, LTB, Germany) with f/10 numerical aperture, 14,000 spectral resolution, and $13 \mathrm{pm}$ wavelength accuracy was employed using its spectral 


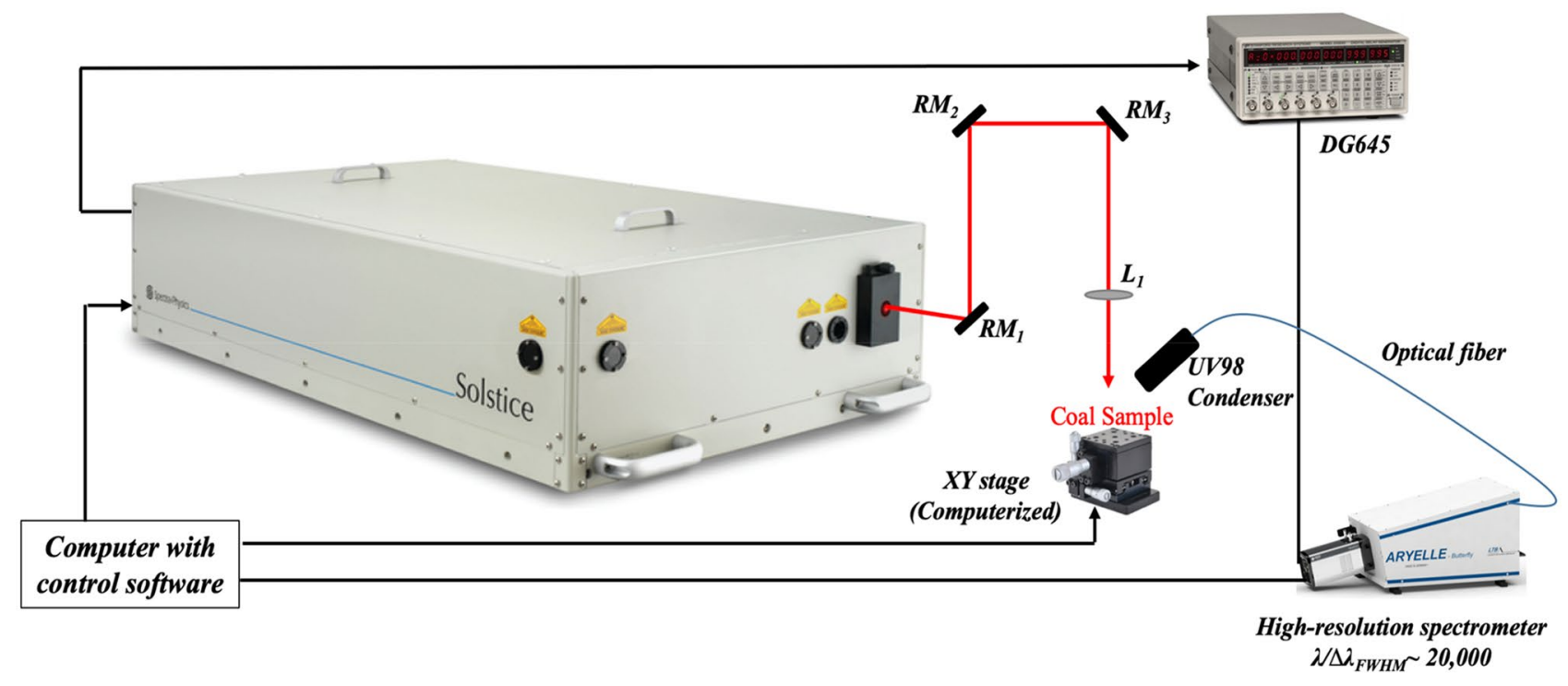

(a)

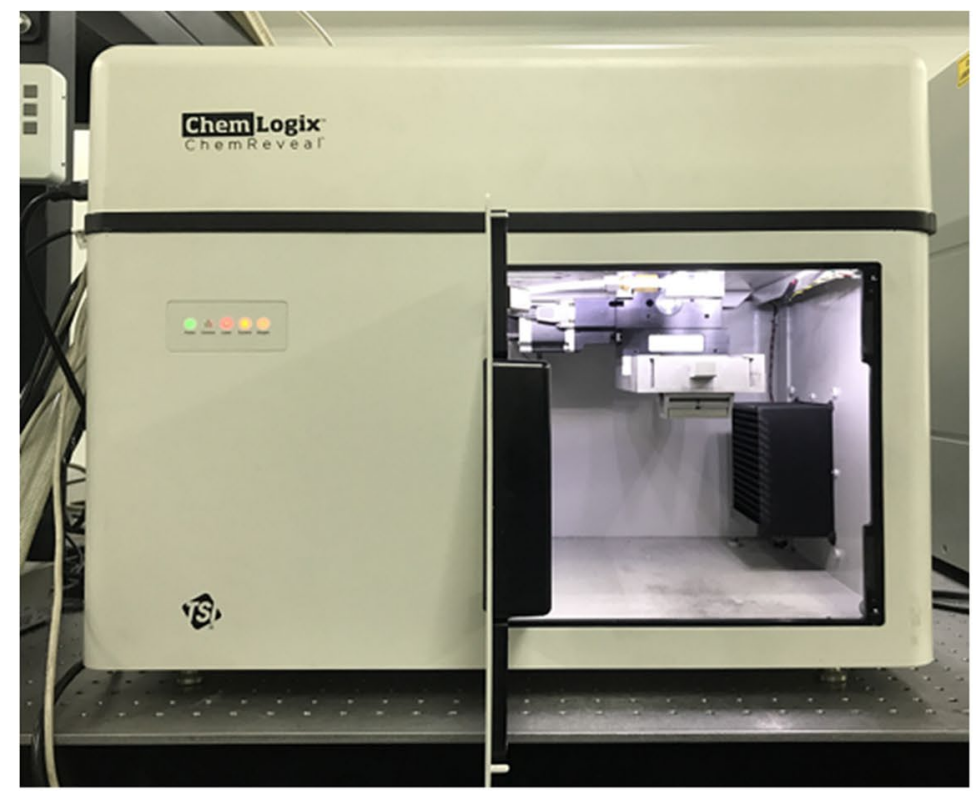

(b)

Figure 13. (a) Schematic diagram (not to scale) of $f$ s-Solstice system under evaluation. RM: ultrafast 730$820 \mathrm{~nm}$ reflecting mirrors; $\mathrm{L}_{1}$ : focusing lens $(f=200 \mathrm{~mm})$; (b) Image of $n s$-Chem system - an industrially-applied ns-LIBS coal analyzer (offline mode of operation). Photographs courtesy of Sahar Sheta. Copyright 2021.

range 192-433 nm. The dark current of the ICCD was subtracted from spectra using Sophi software. For each experimental set, spectrometer was wavelength calibrated using a mercury lamp (DH-3plus, Ocean Tec, USA).

Nanosecond coal analyzer (ns-Chem). An offline LIBS coal analyzer (ChemReveal, TSI, USA) was used to evaluate the fs-LIBS system (Fig. 13b). The instrument (with $50 \times 60.5 \times 50.8 \mathrm{~cm}$ dimensions) encloses: laser source, optics train, sample chamber, and spectrometer. The laser source is a Q-switched Nd: YAG laser emitting pulses at $1064 \mathrm{~nm}$ wavelength with $\sim 8 \mathrm{~ns}$ pulse duration and energies vary over 0 to $200 \mathrm{~mJ} /$ pulse $( \pm 5 \%$ stability) at $1 \mathrm{~Hz}$ repetition rate. Robust opto-mechanical design comprises alignment-free reflecting mirrors and focusing lenses and offers adjustable analysis spot size $(200-800 \mu \mathrm{m})$ with micron-scale precision and control. Sample chamber contains computerized XYZ holder and an optical fiber adjusted $45^{\circ}$ with the laser beam. The spectrometer consists of 7 CCD detectors covering the spectral range 190-940 $\mathrm{nm}$ with a nominal resolution $\sim 0.09 \mathrm{~nm}$ and minimum integration time of $1.05 \mathrm{~ms}$. 


\begin{tabular}{|l|l|l|l|l|}
\hline & $f^{*}$-Solstice & \multicolumn{3}{l|}{ ns-Chem $^{* *}$} \\
\cline { 2 - 5 } & Changeable & Fixed & Changeable & Fixed \\
\hline Laser wavelength, $\mathrm{nm}$ & - & 800 & - & 1064 \\
\hline Pulse frequency, Hz & 1000 & - & - & 1 \\
\hline Pulse duration & - & $45 \mathrm{fs}$ & - & $8 \mathrm{~ns}$ \\
\hline Pulse energy, $\mathrm{mJ}$ & 5.7 & - & 90 & - \\
\hline Warming up time, $\mathrm{h}$ & - & 1.5 & - & 1 \\
\hline Spot diameter, $\mu \mathrm{m}$ & 600 & - & 200 & - \\
\hline Shots per sample & - & 100 & - & 100 \\
\hline Ablation mode & Fs-train of 100 signals & - & - & Single pulse \\
\hline Delay time, $\mu \mathrm{s}$ & 0.4 & - & 1 & - \\
\hline Integration time & $1 \mu \mathrm{s}$ & - & - & $1.05 \mathrm{~ms}$ \\
\hline
\end{tabular}

Table 9. Operation parameters of $f$ s-Solstice and $n s$-Chem systems. ${ }^{\star}$ Optimized for best-achieved: SBR, spectral resolution, and no lines saturation/interferences. ${ }^{* *}$ Factorial design based on best-tested: signals' repeatability and quantitative modeling results.

The aim of this study is to evaluate our fs-LIBS system for coal property analysis application relative to an industrially applied coal analyzer. System evaluation is defined as the comparison of data results to a standard set for the purpose of judging worth of quality ${ }^{71}$ using several objectives to measure effectiveness/performance. In literature, different papers have discussed evaluation of LIBS systems for industrially-related applications. Gaft et al. ${ }^{10}$ evaluated LDS LIBS unit for ash analysis in coal relative to a Coalscan 9500X PGNAA analyzer at Optimum Colliery. Chadwick et al. ${ }^{72}$ evaluated LIBS system for elemental detection of low-ash lignite coal as fuel source. LIBS results were compared relative to standard results set of atomic absorption spectrometry (AAS) at Loy Yang Power. Naes et al. ${ }^{73}$ evaluated LIBS system relative to LA-ICP-MS and $\mu$ XRF systems for the discrimination of automotive glass. Trevizan et al. ${ }^{74}$ evaluated LIBS system for macronutrients in plant materials relative to ICP-OES after wet acid decomposition. At previous studies, LIBS systems under evaluation were operating at best-achieved results relative to optimized conditions of the standard systems. Accordingly, $n s$-Chem system operates in its factorial-adjusted design customized for coal analysis in power plant. While, the operation parameters of $f$ s-Solstice system were selected after an experiment was run using its changeable parameters to achieve best-obtained signal-to-background ratio (SBR), spectral resolution, and to avoid line intensities saturation/interferences. For each fs-pulse energy: 4.9, 5.1, 5.3, 5.7, 5.9, 6.1 and $6.3 \mathrm{~mJ}$, an optimum delay time in the interval of $0-3$ s was chosen. Single, $10,20,50,100$, and 200 pulse trains were tested. The selected operation parameters for the fs-LIBS system are listed in Table 9 with the operation parameters of the ns-coal analyzer. All experiments were performed in an atmospheric environment (Lab. temperature was $\sim 21-23{ }^{\circ} \mathrm{C}$ and humidity was $<20 \%$ ). The data treatment was performed using Matlab R2018b, Origin, and Excel.

Craters morphology. Craters' surface and depth topologies were studied using focused ion beam scanning electron microscopy FIB-SEM (Lyra 3, TESCAN, Czech Republic) and coherence scanning interferometry CSI (Zygo-NexView, Ametek, USA).

Received: 3 May 2021; Accepted: 19 July 2021

Published online: 05 August 2021

\section{References}

1. Franco, A. \& Diaz, A. R. The future challenges for "clean coal technologies": Joining efficiency increase and pollutant emission control. Energy 34, 348-354. https://doi.org/10.1016/j.energy.2008.09.012 (2009).

2. Chang, S., Zhuo, J., Meng, S., Qin, S. \& Yao, Q. Clean coal technologies in China: Current status and future perspectives. Engineering 2, 447-459. https://doi.org/10.1016/J.ENG.2016.04.015 (2016).

3. Sheta, S. et al. Coal analysis by laser-induced breakdown spectroscopy: A tutorial review. J. Anal. Atom. Spectrom. 34, 1047-1082. https://doi.org/10.1039/c9ja00016j (2019).

4. Wang, Z. et al. Recent advances in laser-induced breakdown spectroscopy quantification: From fundamental to data processing. Trends Analyt. Chem. 143, 116385. https://doi.org/10.1016/j.trac.2021.116385 (2021).

5. Romero, C. E. et al. Laser-induced breakdown spectroscopy for coal characterization and assessing slagging propensity. Energy Fuels 24, 510-517. https://doi.org/10.1021/ef900873w (2010).

6. Zhang, L. et al. Development of a coal quality analyzer for application to power plants based on laser-induced breakdown spectroscopy. Spectrochim. Acta B 113, 167-173. https://doi.org/10.1016/j.sab.2015.09.021 (2015).

7. Redoglio, D., Golinelli, E., Musazzi, S., Perini, U. \& Barberis, F. A large depth of field LIBS measuring system for elemental analysis of moving samples of raw coal. Spectrochim. Acta B 116, 46-50. https://doi.org/10.1016/j.sab.2015.11.005 (2016).

8. Redoglio, D. A., Golinelli, E., Musazzi, S., Perini, U. \& Barberis, F. Development of a large depth of field collection optics for on-line laser-induced breakdown spectroscopy applications. Spectrochim. Acta B 123, 179-183. https://doi.org/10.1016/j.sab.2016.07.009 (2016).

9. Gaft, M., Sapir-Sofer, I., Modiano, H. \& Stana, R. Laser induced breakdown spectroscopy for bulk minerals online analyses. Spectrochim. Acta B 62, 1496-1503. https://doi.org/10.1016/j.sab.2007.10.041 (2007).

10. Gaft, M., Dvir, E., Modiano, H. \& Schone, U. Laser induced breakdown spectroscopy machine for online ash analyses in coal. Spectrochim. Acta B 63, 1177-1182. https://doi.org/10.1016/j.sab.2008.06.007 (2008). 
11. Sheta, S. et al. Insights into enhanced repeatability of femtosecond laser-induced plasmas. ACS Omega 5, 30425-30435. https:// doi.org/10.1021/acsomega.0c03636 (2020).

12. Zhang, B., He, M., Hang, W. \& Huang, B. Minimizing matrix effect by femtosecond laser ablation and ionization in elemental determination. Anal. Chem. 85, 4507-4511. https://doi.org/10.1021/ac400072j (2013).

13. Zorba, V., Mao, X. \& Russo, R. E. Ultrafast laser induced breakdown spectroscopy for high spatial resolution chemical analysis. Spectrochimica Acta B 66, 189-192. https://doi.org/10.1016/j.sab.2010.12.008 (2011).

14. Schaffer, C. B., Brodeur, A. \& Mazur, E. Laser-induced breakdown and damage in bulk transparent materials induced by tightly focused femtosecond laser pulses. Meas. Sci. Technol. 12, 1784-1794. https://doi.org/10.1088/0957-0233/12/11/305 (2001).

15. Stuart, B. C. et al. Nanosecond-to-femtosecond laser-induced breakdown in dielectrics. Phys. Rev. B 53, 1749-1761. https://doi. org/10.1103/PhysRevB.53.1749 (1996).

16. Hemalaxmi, R., Aparna, N., Vasa, N. J. \& Seshadri, S. Nanosecond and femtosecond laser induced breakdown spectroscopic studies of coal and ash. in 2018 Conference on Lasers and Electro-Optics Pacific Rim (CLEO-PR). (2018).

17. Jian, H., Jingqi, L., Huan, Y. \& Xiangdong, C. Quantitative analysis on coal calorific value using nanosecond, femtosecond, and dual-pulse laser-induced breakdown spectroscopy. Laser Optoelectron. Progress 56, 193002. https://doi.org/10.3788/lop56.193002 (2019).

18. Yuan, T. et al. Coal property analysis using laser-induced breakdown spectroscopy. J. Anal. Atom. Spectrom. 28, 1045-1053. https:// doi.org/10.1039/C3JA50097G (2013).

19. Lu, L., Sahajwalla, V., Kong, C. \& Harris, D. Quantitative X-ray diffraction analysis and its application to various coals. Carbon 39, 1821-1833. https://doi.org/10.1016/S0008-6223(00)00318-3 (2001).

20. NIST Atomic Spectra Database. https://www.nist.gov/pml/atomic-spectra-database.

21. Yao, S. et al. Extracting coal ash content from laser-induced breakdown spectroscopy (LIBS) spectra by multivariate analysis. Appl. Spectrosc. 65, 1197-1201. https://doi.org/10.1366/10-06190 (2011).

22. Hou, Z. et al. A hybrid quantification model and its application for coal analysis using laser induced breakdown spectroscopy. J. Anal. Atom. Spectrom. 31, 722-736. https://doi.org/10.1039/C5JA00475F (2016).

23. Fu, Y. T. et al. Mechanism of signal uncertainty generation for laser-induced breakdown spectroscopy. Front. Phys. 16, 22502. https://doi.org/10.1007/s11467-020-1006-0 (2021).

24. Yan, C. et al. A novel hybrid feature selection strategy in quantitative analysis of laser-induced breakdown spectroscopy. Anal. Chim. Acta 1080, 35-42. https://doi.org/10.1016/j.aca.2019.07.012 (2019).

25. Adler, J. \& Parmryd, I. Quantifying colocalization by correlation: The Pearson correlation coefficient is superior to the Mander's overlap coefficient. Cytometry A 77A, 733-742. https://doi.org/10.1002/cyto.a.20896 (2010).

26. Gornushkin, I. B., Mueller, M., Panne, U. \& Winefordner, J. D. Insights into linear and rank correlation for material identification in laser-induced breakdown spectroscopy and other spectral techniques. Appl. Spectrosc. 62, 542-553. https://doi.org/10.1366/ 000370208784344505 (2008).

27. Gornushkin, I. B., Panne, U. \& Winefordner, J. D. Linear correlation for identification of materials by laser induced breakdown spectroscopy: Improvement via spectral filtering and masking. Spectrochim. Acta B 64, 1040-1047. https://doi.org/10.1016/j.sab. 2009.07.038 (2009).

28. Lentjes, M., Dickmann, K. \& Meijer, J. Calculation and optimization of sample identification by laser induced breakdown spectroscopy via correlation analysis. Spectrochim. Acta B 62, 56-62. https://doi.org/10.1016/j.sab.2006.11.009 (2007).

29. Chirinos, J. et al. Remote isotope detection and quantification using femtosecond filament-laser ablation molecular isotopic spectrometry. Spectrochim. Acta B 179, 106117. https://doi.org/10.1016/j.sab.2021.106117 (2021).

30. Zhang, Q. Y. et al. Determination of vibrational and rotational temperatures in highly constricted nitrogen plasmas by fitting the second positive system of N2 molecules. AIP Adv. 5, 057158. https://doi.org/10.1063/1.4921916 (2015).

31. Ventura, L. R. \& Fellows, C. E. The N2 second positive (C3Пu $\rightarrow$ B3Пg) system reviewed: Improved data and analysis. J. Quant. Spectrosc. Radiat. Transf. 239, 106645. https://doi.org/10.1016/j.jqsrt.2019.106645 (2019).

32. Yamanouchi, K. The next frontier. Science 295, 1659. https://doi.org/10.1126/science.1068449 (2002).

33. Serrano, J., Moros, J. \& Javier Laserna, J. Molecular signatures in femtosecond laser-induced organic plasmas: Comparison with nanosecond laser ablation. Phys. Chem. Chem. Phys. 18, 2398-2408. https://doi.org/10.1039/C5CP06456B (2016).

34. Baudelet, M. et al. Spectral signature of native CN bonds for bacterium detection and identification using femtosecond laserinduced breakdown spectroscopy. Appl. Phys. Lett. 88, 063901. https://doi.org/10.1063/1.2170437 (2006).

35. Zhao, Y. L., Li, G. G., Hou, H. M., Shi, J. C. \& Luo, S. N. CN and C2 formation mechanisms in fs-laser induced breakdown of nitromethane in Ar or N2 atmosphere. J. Hazard. Mater. 393, 122396. https://doi.org/10.1016/j.jhazmat.2020.122396 (2020).

36. Danylo, R. et al. Formation dynamics of excited neutral nitrogen molecules inside femtosecond laser filaments. Phys. Rev. Lett. 123, 243203. https://doi.org/10.1103/PhysRevLett.123.243203 (2019).

37. Xu, H., Cheng, Y., Chin, S.-L. \& Sun, H.-B. Femtosecond laser ionization and fragmentation of molecules for environmental sensing. Laser Photon. Rev. 9, 275-293. https://doi.org/10.1002/lpor.201400208 (2015).

38. Yao, S. et al. Analysis of spectral properties for coal with different volatile contents by laser-induced breakdown spectroscopy. Spectrochim. Acta B 149, 249-255. https://doi.org/10.1016/j.sab.2018.09.002 (2018).

39. Freeman, J. R., Harilal, S. S., Diwakar, P. K., Verhoff, B. \& Hassanein, A. Comparison of optical emission from nanosecond and femtosecond laser produced plasma in atmosphere and vacuum conditions. Spectrochim. Acta B 87, 43-50. https://doi.org/10. 1016/j.sab.2013.05.011 (2013).

40. LaHaye, N. L., Phillips, M. C., Duffin, A. M., Eiden, G. C. \& Harilal, S. S. The influence of ns- and fs-LA plume local conditions on the performance of a combined LIBS/LA-ICP-MS sensor. J. Anal. Atom. Spectrom. 31, 515-522. https://doi.org/10.1039/C5JA0 0317B (2016).

41. Finkelman, R. B., Dai, S. \& French, D. The importance of minerals in coal as the hosts of chemical elements: A review. Int. J. Coal Geol. 212, 103251. https://doi.org/10.1016/j.coal.2019.103251 (2019).

42. Dong, M. et al. Application of LIBS for direct determination of volatile matter content in coal. J. Anal. Atom. Spectrom. 26, 2183-2188. https://doi.org/10.1039/C1JA10109A (2011).

43. Vieth, W. \& Huneke, J. C. Relative sensitivity factors in glow discharge mass spectrometry. Spectrochim. Acta B 46, 137-153. https:// doi.org/10.1016/0584-8547(91)80017-W (1991).

44. Wilson, R. G. \& Novak, S. W. Systematics of secondary-ion-mass spectrometry relative sensitivity factors versus electron affinity and ionization potential for a variety of matrices determined from implanted standards of more than 70 elements. J. Appl. Phys. 69, 466-474. https://doi.org/10.1063/1.347687 (1991).

45. Di Sabatino, M., Dons, A. L., Hinrichs, J. \& Arnberg, L. Determination of relative sensitivity factors for trace element analysis of solar cell silicon by fast-flow glow discharge mass spectrometry. Spectrochim. Acta B 66, 144-148. https://doi.org/10.1016/j.sab. 2011.01.004 (2011).

46. Noda, M., Deguchi, Y., Iwasaki, S. \& Yoshikawa, N. Detection of carbon content in a high-temperature and high-pressure environment using laser-induced breakdown spectroscopy. Spectrochim. Acta B 57, 701-709. https://doi.org/10.1016/S0584-8547(01) 00403-7 (2002).

47. Gross, D. S., Gälli, M. E., Silva, P. J. \& Prather, K. A. Relative sensitivity factors for alkali metal and ammonium cations in singleparticle aerosol time-of-flight mass spectra. Anal. Chem. 72, 416-422. https://doi.org/10.1021/ac990434g (2000). 
48. Povarnitsyn, M. E., Itina, T. E., Sentis, M., Khishchenko, K. V. \& Levashov, P. R. Material decomposition mechanisms in femtosecond laser interactions with metals. Phys. Rev. B 75, 235414. https://doi.org/10.1103/PhysRevB.75.235414 (2007).

49. Wu, D. et al. Dynamic characteristics of multi-charged ions emitted from nanosecond laser produced molybdenum plasmas. J. Anal. Atom. Spectrom. 35, 767-775. https://doi.org/10.1039/C9JA00411D (2020).

50. Kanesue, T., Kumaki, M., Ikeda, S. \& Okamura, M. Low charge state heavy ion production with sub-nanosecond laser. Rev. Sci. Instrum. 87, 02A916. https://doi.org/10.1063/1.4935625 (2015).

51. Liu, C. et al. Nanosecond and femtosecond laser ablation of brass: Particulate and ICPMS measurements. Anal. Chem. 76, 379-383. https://doi.org/10.1021/ac035040a (2004).

52. Carlson, G. A. Computer simulation of the molecular structure of bituminous coal. Energy Fuels 6, 771-778. https://doi.org/10. 1021/ef00036a012 (1992).

53. Chichkov, B. N., Momma, C., Nolte, S., von Alvensleben, F. \& Tünnermann, A. Femtosecond, picosecond and nanosecond laser ablation of solids. Appl. Phys. A 63, 109-115. https://doi.org/10.1007/BF01567637 (1996).

54. Furusawa, K., Takahashi, K., Kumagai, H., Midorikawa, K. \& Obara, M. Ablation characteristics of Au, Ag, and Cu metals using a femtosecond Ti:sapphire laser. Appl. Phys. A 69, S359-S366. https://doi.org/10.1007/s003390051417 (1999).

55. Vorobyev, A. Y. \& Guo, C. Enhanced energy coupling in femtosecond laser-metal interactions at high intensities. Opt. Express 14, 13113-13119. https://doi.org/10.1364/OE.14.013113 (2006).

56. Vorobyev, A. Y. \& Guo, C. Direct observation of enhanced residual thermal energy coupling to solids in femtosecond laser ablation. Appl. Phys. Lett. 86, 011916. https://doi.org/10.1063/1.1844598 (2004).

57. Fujimoto, J. G., Liu, J. M., Ippen, E. P. \& Bloembergen, N. Femtosecond laser interaction with metallic tungsten and nonequilibrium electron and lattice temperatures. Phys. Rev. Lett. 53, 1837-1840. https://doi.org/10.1103/PhysRevLett.53.1837 (1984).

58. Byskov-Nielsen, J., Savolainen, J.-M., Christensen, M. S. \& Balling, P. Ultra-short pulse laser ablation of metals: Threshold fluence, incubation coefficient and ablation rates. Appl. Phys. A 101, 97-101. https://doi.org/10.1007/s00339-010-5766-1 (2010).

59. Shaheen, M. E., Gagnon, J. E. \& Fryer, B. J. Femtosecond laser ablation behavior of gold, crystalline silicon, and fused silica: A comparative study. Laser Phys. 24, 106102. https://doi.org/10.1088/1054-660x/24/10/106102 (2014).

60. Huang, M., Zhao, F., Cheng, Y., Xu, N. \& Xu, Z. Origin of laser-induced near-subwavelength ripples: Interference between surface plasmons and incident laser. ACS Nano 3, 4062-4070. https://doi.org/10.1021/nn900654v (2009).

61. Höhm, S., Rosenfeld, A., Krüger, J. \& Bonse, J. Area dependence of femtosecond laser-induced periodic surface structures for varying band gap materials after double pulse excitation. Appl. Surf. Sci. 278, 7-12. https://doi.org/10.1016/j.apsusc.2012.10.188 (2013).

62. Ashkenasi, D., Lorenz, M., Stoian, R. \& Rosenfeld, A. Surface damage threshold and structuring of dielectrics using femtosecond laser pulses: The role of incubation. Appl. Surf. Sci. 150, 101-106. https://doi.org/10.1016/S0169-4332(99)00228-7 (1999).

63. Zhao, D. et al. Ablation mass features in multi-pulses femtosecond laser ablate molybdenum target. Nucl. Instrum. Methods Phys. Res., Sect. B 418, 54-59. https://doi.org/10.1016/j.nimb.2017.12.030 (2018).

64. Vorobyev, A. Y. \& Guo, C. Reflection of femtosecond laser light in multipulse ablation of metals. J. Appl. Phys. 110, 043102. https:// doi.org/10.1063/1.3620898 (2011).

65. Bonse, J., Wrobel, J. M., Krüger, J. \& Kautek, W. Ultrashort-pulse laser ablation of indium phosphide in air. Appl. Phys. A 72, 89-94. https://doi.org/10.1007/s003390000596 (2001).

66. Griem, H. R. in Fast Electrical and Optical Measurements: Volume I: Current and Voltage Measurements/Volume II: Optical Measurements (eds J. E. Thompson \& L. H. Luessen) 885-910 (Springer, 1986).

67. Unnikrishnan, V. K. et al. Measurements of plasma temperature and electron density in laser-induced copper plasma by timeresolved spectroscopy of neutral atom and ion emissions. Pramana 74, 983-993. https://doi.org/10.1007/s12043-010-0089-5 (2010).

68. Wang, M. et al. Multiscale visualization of colloidal particle lens array mediated plasma dynamics for dielectric nanoparticle enhanced femtosecond laser-induced breakdown spectroscopy. Anal. Chem. 91, 9952-9961. https://doi.org/10.1021/acs.analc hem.9b01686 (2019).

69. Labutin, T. A., Lednev, V. N., Ilyin, A. A. \& Popov, A. M. Femtosecond laser-induced breakdown spectroscopy. J. Anal. Atom. Spectrom. 31, 90-118. https://doi.org/10.1039/c5ja00301f (2016).

70. Gurevich, E. L. \& Hergenroeder, R. Femtosecond laser-induced breakdown spectroscopy: Physics, applications, and perspectives. Appl. Spectrosc. 61, 233A-242A. https://doi.org/10.1366/000370207782217824 (2007).

71. Hamilton, S. \& Chervany, N. L. Evaluating information system effectiveness-part i: Comparing evaluation approaches. MIS Q. 5, 55-69. https://doi.org/10.2307/249291 (1981).

72. Chadwick, B. L. \& Body, D. Development and commercial evaluation of laser-induced breakdown spectroscopy chemical analysis technology in the coal power generation industry. Appl. Spectrosc. 56, 70-74 (2002).

73. Naes, B. E., Umpierrez, S., Ryland, S., Barnett, C. \& Almirall, J. R. A comparison of laser ablation inductively coupled plasma mass spectrometry, micro X-ray fluorescence spectroscopy, and laser induced breakdown spectroscopy for the discrimination of automotive glass. Spectrochim. Acta B 63, 1145-1150. https://doi.org/10.1016/j.sab.2008.07.005 (2008).

74. Trevizan, L. C. et al. Evaluation of laser induced breakdown spectroscopy for the determination of macronutrients in plant materials. Spectrochim. Acta B 63, 1151-1158. https://doi.org/10.1016/j.sab.2008.08.005 (2008).

\section{Acknowledgements}

This work is supported by National Natural Science Foundation of China (No. 61675110) and National Key Research and Development Program Key Projects of China (No. 2016YFC0302102). Authors are grateful to Mrs. Wang Rong and Mrs. Liang Yimai for SEM images and surface profile measurements.

\section{Author contributions}

S.S. conceived the idea, designed the experiment, and analyzed the data under the guidance of Z.W. S.S. wrote the original manuscript and Z.W. discussed it. Z.H. and Y.W. reviewed the original manuscript. All authors have given approval to the final version of the manuscript.

\section{Competing interests}

The authors declare no competing interests.

\section{Additional information}

Correspondence and requests for materials should be addressed to S.S. or Z.W.

Reprints and permissions information is available at www.nature.com/reprints.

Publisher's note Springer Nature remains neutral with regard to jurisdictional claims in published maps and institutional affiliations. 
(c) (i) Open Access This article is licensed under a Creative Commons Attribution 4.0 International cc) License, which permits use, sharing, adaptation, distribution and reproduction in any medium or format, as long as you give appropriate credit to the original author(s) and the source, provide a link to the Creative Commons licence, and indicate if changes were made. The images or other third party material in this article are included in the article's Creative Commons licence, unless indicated otherwise in a credit line to the material. If material is not included in the article's Creative Commons licence and your intended use is not permitted by statutory regulation or exceeds the permitted use, you will need to obtain permission directly from the copyright holder. To view a copy of this licence, visit http://creativecommons.org/licenses/by/4.0/.

(C) The Author(s) 2021 